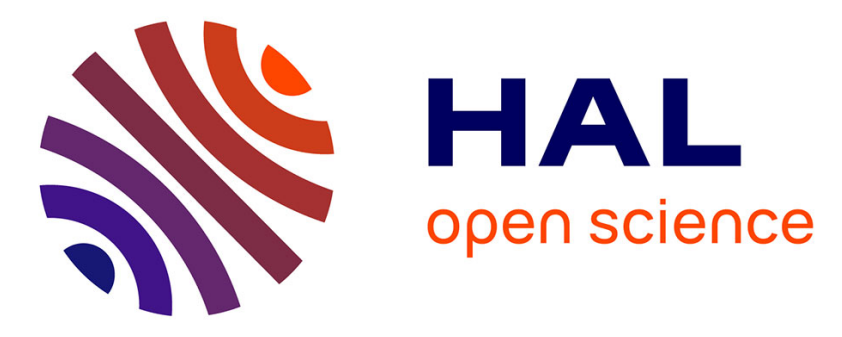

\title{
Bundle-specific tractography with incorporated anatomical and orientational priors
}

Francois Rheault, Etienne St-Onge, Jasmeen Sidhu, Klaus Maier-Hein, Nathalie Tzourio-Mazoyer, Laurent Petit, Maxime Descoteaux

\section{- To cite this version:}

Francois Rheault, Etienne St-Onge, Jasmeen Sidhu, Klaus Maier-Hein, Nathalie Tzourio-Mazoyer, et al. Bundle-specific tractography with incorporated anatomical and orientational priors. NeuroImage, 2019, 186, pp.382-398. 10.1016/j.neuroimage.2018.11.018 . hal-02063638

\section{HAL Id: hal-02063638 \\ https://hal.science/hal-02063638}

Submitted on 13 Nov 2020

HAL is a multi-disciplinary open access archive for the deposit and dissemination of scientific research documents, whether they are published or not. The documents may come from teaching and research institutions in France or abroad, or from public or private research centers.
L'archive ouverte pluridisciplinaire HAL, est destinée au dépôt et à la diffusion de documents scientifiques de niveau recherche, publiés ou non, émanant des établissements d'enseignement et de recherche français ou étrangers, des laboratoires publics ou privés. 


\title{
Bundle-Specific Tractography with incorporated anatomical and orientational priors
}

\author{
Francois Rheault ${ }^{\mathrm{a}, *}$, Etienne St-Onge ${ }^{\mathrm{a}}$, Jasmeen Sidhu ${ }^{\mathrm{a}}$, Klaus Maier-Hein ${ }^{\mathrm{c}}$, Nathalie \\ Tzourio-Mazoyer $^{\mathrm{b}}$, Laurent Petit ${ }^{\mathrm{b}}$, Maxime Descoteaux ${ }^{\mathrm{a}}$ \\ ${ }^{a}$ Sherbrooke Connectivity Imaging Laboratory (SCIL), Université de Sherbrooke, Canada \\ ${ }^{b}$ Groupe d'Imagerie Neurofonctionnelle, IMN, UMR5293, CNRS, CEA, Université de Bordeaux, \\ France \\ ${ }^{c}$ Division of Medical Image Computing, German Cancer Research Center (DKFZ), Heidelberg, \\ Germany
}

\begin{abstract}
Anatomical white matter bundles vary in shape, size, length, and complexity, making diffusion MRI tractography reconstruction of some bundles more difficult than others. As a result, bundles reconstruction often suffers from a poor spatial extent recovery. To fillup the white matter volume as much and as best as possible, millions of streamlines can be generated and filtering techniques applied to address this issue. However, well-known problems and biases are introduced such as the creation of a large number of false positives and over-representation of easy-to-track parts of bundles and under-representation of hard-to-track.

To address these challenges, we developed a Bundle-Specific Tractography (BST) algorithm. It incorporates anatomical and orientational prior knowledge during the process of streamline tracing to increase reproducibility, sensitivity, specificity and efficiency when reconstructing certain bundles of interest. BST outperforms classical deterministic, probabilistic, and global tractography methods. The increase in anatomically plausible streamlines, with larger spatial coverage, helps to accurately represent the full shape of bundles, which could greatly enhance and robustify tract-based and connectivity-based neuroimaging studies.
\end{abstract}

Keywords: Diffusion MRI, White Matter, Tractography, Tractometry, Connectivity

\section{1. Introduction}

Tractography algorithms are currently challenged by several important open issues and biases that need to be addressed to improve brain connectivity mapping [Jones, 2010; Jbabdi and Johansen-Berg, 2011; Maier-Hein et al., 2017]. In Maier-Hein et al. [2017], it was recently shown that whole-brain tractography can recover most existing true positive bundles but that these are poorly recovered in terms of spatial extent. In the

*2500, boul. de l'Université, Sherbrooke (Québec) Canada, J1K 2R1

Email address: Francois.M.Rheault@USherbrooke.ca (Francois Rheault)

Preprint submitted to Neuroimage

November 13, 2018 
simulations of 25 human-like synthetic white matter (WM) bundles, an average spatial extent recovery of only $30 \%$ is reported [Maier-Hein et al., 2017, Fig. 3]. We propose to term this tractography issue as the "poor-spatial-extent" challenge. Techniques that have better spatial extent recovery always generate more invalid streamlines, and thus fall into the usual sensitivity-specificity curse. In the current context, the notion of invalid streamlines refers to anatomically improbable streamlines, meaning their path is not reflective of known WM pathways. The quality of WM bundle reconstruction using tractography is influenced by many variables: i) global geometry of the bundle like curvature, thickness, length, ii) local crossing regions it traverses, position of the bundle with respect to other bundles, and iii) extent and shape of cortical endpoints. As a result, some WM bundles are harder-to-track than others, which leads to the poor-spatial-extent challenge.

Why are some bundles more difficult to reconstruct than others? Complex local fiber regions, even if well recovered locally in terms of fiber orientation distributions, can lead to invalid tractography streamlines that respect the underlying local diffusion signal but make clear invalid pathways at the global brain level [Maier-Hein et al., 2017]. Despite the fact that almost all WM regions contain crossing fiber populations [Jeurissen et al., 2013], some are well-know pitfalls for tractography methods and could be tackled with better prior information. As an illustration, the centrum semiovale, as seen in Figure 1 and Figure 2, was and still is a major difficulty for tractography algorithms. Three major WM pathways: i) the pyramidal tract, ii) the mid-body of the corpus callosum (anterior and posterior), and iii) the arching streamlines connecting the frontal to the temporal lobes, are crossing almost orthogonally in the most ventral part. At such an intersection, even with multiple fiber techniques, tractography algorithms most often follow the easiest path available. Bundles can seem to cover a large spatial extent and have an appropriate amount of fanning when millions of streamlines are used. However, the density is far from uniform within a bundle, even if it cannot be perceived visually, as seen in Figure 2. The amount of crossing regions, overall length and curvature of the bundle will influence the probability of a streamlines to reach its expected destination. Moreover, it is common to observe streamlines abruptly changing direction to either switch to a different bundle or follow an erroneous path, but end up in the right location. Crossing can become even harder to distinguish as the main direction of the fiber populations kiss or come into a bottleneck [Maier-Hein et al., 2017, Fig.7].

A partially reconstructed WM bundle could lead to erroneous interpretations and
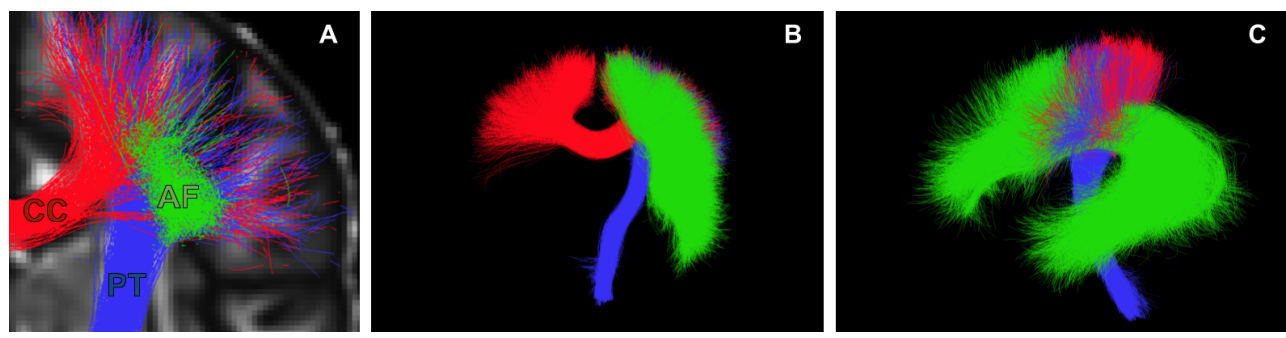

Figure 1: Crossing region of the Corpus Callosum (red), the Arcuate Fasciculus (green) and the Pyramidal Tract (blue) in the centrum semiovale. A) Coronal cross-section, B) coronal 3D view and C) sagittal $3 \mathrm{D}$ view. 

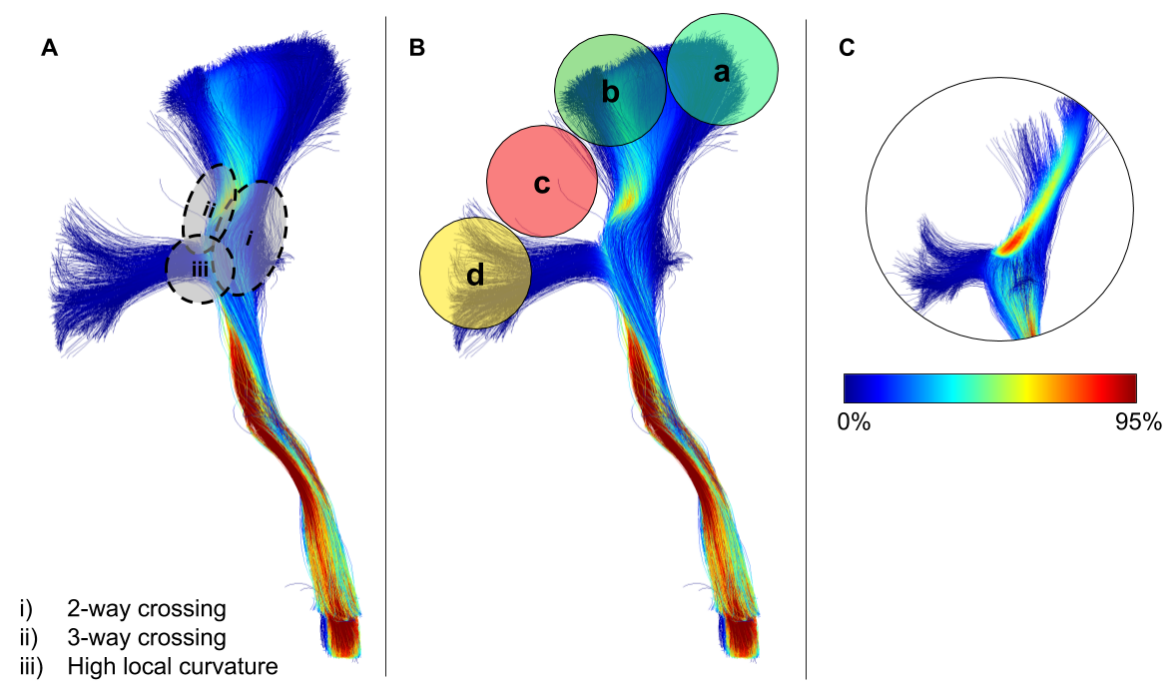

Figure 2: Reconstruction of pyramidal tract (PT) streamlines illustrating some of the challenges of tractography, seen on panel A, i) 2-way crossing ii) 3-way crossing, and iii) high curvature. In panel B, regions $a$ and $b$ encounter less difficulties in reconstruction due to only being subject to a 2-way crossing limitation, as illustrated in Panel A (i), leading to a higher proportion of streamlines reaching the cortex in these general regions. Conversely, region $c$ in Panel B encounters 2 and 3 way crossings (Panel A (i) and (ii)), whereas, region $d$ in Panel B encounters 2-way crossing and high-curvature limitations (Panel A (i) and (iii)), leading to a lower proportion of streamlines successfully reaching the cortex. Streamline coloring represents the local streamlines density (at the voxel-level), segments in red shows regions where a large proportion of streamlines pass through a bottleneck as opposed to segments in blue. Panel C illustrates that the majority of streamlines traverse through the core of the PT.

conclusions. At the tract-based analysis level [Dayan et al., 2016; Cousineau et al., 2017; Yeatman et al., 2012, 2018], the spatial extent of bundles (in $\mathrm{cm}^{3}$ ) is a measurement often used to investigate morphological group differences, such as atrophy or asymmetry [Catani et al., 2007; Song et al., 2014; Chenot et al., 2018]. At the connectome-level, the number of streamlines in WM bundles connecting all possible pairs of cortical regions is usually used [Sotiropoulos and Zalesky, 2017]. However, because of a combination of may have easier-to-track bundles than other subjects, which results in WM bundles that fill-up in spatial extent before others and thus have artificially larger number of tractography with millions of streamlines followed by manual, clustering or advanced filtering techniques, 2) a tailored ROI-based tractography.

Whole-brain tractography followed by filtering, clustering or manual dissection. In the first strategy, millions of streamlines are typically reconstructed using a whole-brain deterministic, probabilistic, or global fiber tracking algorithms to fill-up the whole WM volume and be able to capture a large spatial extent of all bundles [Wassermann et al., 2016; Jeurissen et al., 2017; Moyer et al., 2018; Calamante et al., 2015; Smith et al., 2015b]. With more streamlines, a larger extent of bundles is reconstructed (higher sensitivity) but also more false positive bundles created (lower specificity) [Côté et al., 2013; 
Maier-Hein et al., 2017], thus leading to the sensitivity-specificity curse mentioned before. Generation of millions streamlines is often considered a brute force approach with a heavy computational burden. All iterations leading to false positive are considered wasted. Reducing such iterations increases the computational efficiency of a method, meaning that less effort is required while maintaining an acceptable sensitivity-specificity ratio.

Moreover, when millions of streamlines are desired in a whole brain tractogram, millions of seeds and hundreds of millions of 3D points are generated, which can be an important computational and memory limitation [Rheault et al., 2017]. In the case of a specific tract-based application, where only a single to a few WM bundles are desired, this whole brain strategy can be sub-optimal both in terms of bundle reconstruction quality and computational efforts. Increasing the number of streamlines to $10 \mathrm{M}$ or $100 \mathrm{M}$ would fill-up more of this hard-to-track fanning space, but would potentially never reach full extent coverage. Generating more streamlines will artificially increase the density of the central, easy-to-track part of the bundle and generate a large number of false positive streamlines. Advanced filtering techniques such as SIFT, LIFE, COMMIT [Smith et al., 2015a; Pestilli et al., 2014; Daducci et al., 2015] are able to filter some false positives and reduce the density bias by making sure streamlines explain the desired diffusion signal, but at the cost of removing some hard-to-track valid streamlines as well [Maier-Hein et al., 2017].

ROI-based tractography. In the second strategy, a ROI-based tractography strategy uses different masks, filtering ROI pass-ways and varying tractography parameters (step size, curvature, anisotropy thresholds) [Chamberland et al., 2014, 2017] to enhance the quality of results based on neuroanatomical prior knowledge. A ROI-based seeding strategy is an efficient way to quickly target a bundle of interest by removing unnecessary computations needed in whole brain tractography. Such strategy has already been used in multiple research projects to avoid generating unwanted streamlines [Basser et al., 2000; Catani et al., 2002; Mori and van Zijl, 2002; Behrens et al., 2007; Ghaziri et al., 2015; Renauld et al., 2016; Rozanski et al., 2017; Chamberland et al., 2017], mainly using manual delineations, or ROIs obtained from a segmentation tool such as FreeSurfer [Desikan et al., 2006], or the JHU template [Mori et al., 2005].

Here, ROI-based tractography can be more efficient but not necessarily lead to better spatial extent recovery. The poor-spatial-extent problem is temporarily "hidden" because one can now afford to launch millions of seeds just to reconstruct a single bundle. However, the same issues illustrated in Figure 2 remain, i.e. hard-to-track fanning regions are hard to reach and easy-to-track regions are amplified. Also, because of the ROI-based filtering process, all the false positive streamlines generated are thrown out and excluded from the result, but, computational efforts are still deployed to track them. Hence, overall, a ROI-based approach still suffers from poor efficiency, incomplete spatial-coverage and a similar underlying trade-off between sensitivity and specificity.

To address these issues, tractography needs the injection of more information and priors into the streamline tracing process itself. In Maier-Hein et al. [2017], it was clearly shown that following local orientations alone cannot resolve all the potential ambiguity and biases of fiber tracking. Bottlenecks are not tractable using directional information alone.

Several approaches have been proposed to inject more prior knowledge. An automated method called TRACULA [Yendiki et al., 2011] was proposed to include anatomical 
priors from anatomical labeling. Pathway labels are used to initialize and constrain the probabilistic tractography algorithm. However, this approach uses a conservative reconstruction model (FACT, [Mori et al., 1999]) and strict anatomical definitions as training sets, which limits the spatial extent of bundles. Further, probability maps are computed instead of streamlines potentially limiting tract-based analyses. More recently, Wasserthal et al. [2018a] proposed a method to directly segment the volume occupied by bundle of interests. However this method does not rely on tractography and does not produce streamlines. Features of the raw diffusion signal are learned and regions of interests segmented where the characteristics of the signal match with bundles of interests.

On the other hand, global tractography algorithms [Kreher et al., 2008; Mangin et al., 2013; Christiaens et al., 2015; Neher et al., 2012] can sometimes overcome difficult local crossing regions, but resulting tractograms do not always represent the expected anatomical complexity of fasciculi. Global tracking algorithms are promising but are known to have their share of open challenges such as heavy computational needs, the generation of a large number of broken streamlines, and difficulty to impose anatomical priors [Maier-Hein et al., 2017; Jeurissen et al., 2017].

Other approaches exist to overcome fiber crossing difficulties. Chamberland et al. [2017] proposed a magnetic tracking (MAGNET) tool to manually influence directions in strategic regions, improving the reconstruction of the optic radiations bundle. MAGNET essentially pulls tractography towards the occipital lobe with an orientation prior, allowing fiber tracking to perform a "U-turn"-like reconstruction needed to fully reconstruct Meyer's loop. Moreover, Dhollander et al. [2014] also suggested a method to sharpen orientations based on streamline distributions, called Track-Orientation Distributions (TOD). TOD can help enhance tractography to cover longer distances along WM structures and address some of the biases of fiber tracking. However, since streamlines density is not uniform across a tractogram and does not represent the underlying anatomy, using such a method iteratively across WM will increase the density bias in easy-to-track regions. More recently, Wasserthal et al. [2018b] proposed an approach using bundle-specific orientation maps learned from multiple datasets to select a single fiber orientation distribution (FOD) peak in each voxel, thus improving reconstruction of bundles by simplifying regions with complex WM configuration. To the best of our knowledge, these techniques are the only existing methods trying to inject orientational prior knowledge into the tractography process. Other approaches have proposed to include an orientation-based regularization term in the local reconstruction of fiber orientations, but to our knowledge, not in the tractography procedure itself [Reisert and Kiselev, 2011; Portegies et al., 2015].

Tractography is therefore currently blind to the anatomy and does not have access to the anatomical knowledge accumulated over the past several hundred years by the neuroanatomy community. This neuroanatomical information, when available, could enhance tractography. For instance, priors could capture the "where" and "how" of bundles. That is where should the origin and target region of bundles be, and how difficult crossing, fanning, bending regions should be traversed. Our novel Bundle-Specific Tractography (BST) algorithm is designed to directly incorporate these anatomical and orientational priors in the tractography process itself. The main contribution is a novel methodology enhancing local fiber orientation distribution based on WM bundle priors coming from templates of streamlines. BST is fully automatic and yields better spatial 
coverage, increases quality in the fanning extents and produces more plausible shape reconstructions of bundles in the centrum semiovale.

\section{Methods}

\subsection{Bundle-Specific Tractography}

Our novel bundle-specific tractography (BST) approach is composed of three steps. For each bundle of interest to be reconstructed, one needs to:

1. Build a template of streamlines that represents the shape and position of each bundle, covering as much geometric variability as possible (see first row of Figure 3 and Figure 4).

2. Build the anatomical priors by incorporating dilated versions of endpoints and spatial coverage maps defined from the template of streamlines in 1) (see second row of Figure 3).

3. Build the orientation priors by generating a field of enhanced fiber orientation distrubtions (E-FOD) from the track-orientation distribution of the template of streamlines in 1) (see Figure 4).

Our BST approach can be applied to any bundle of interest with the previous 3 steps. We now detail each of these steps before describing the datasets and how it was applied to bundles intersecting in the centrum semiovale seen in Figure 1.
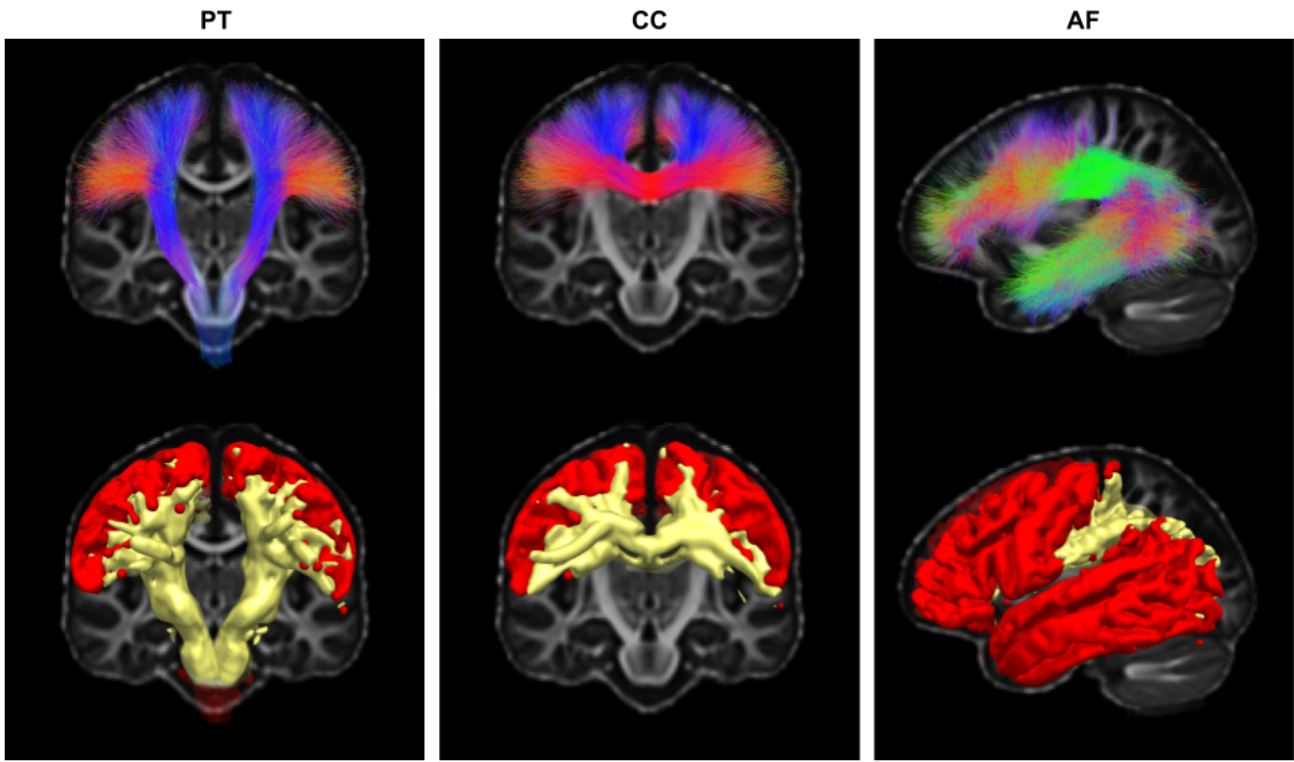

Figure 3: The first row shows streamlines from the template on top of the FA template. In the second row tracking masks (yellow) and seeding endpoints masks (red), automatically extracted from the template of streamlines of the first row. 
a) 1 template (common space)

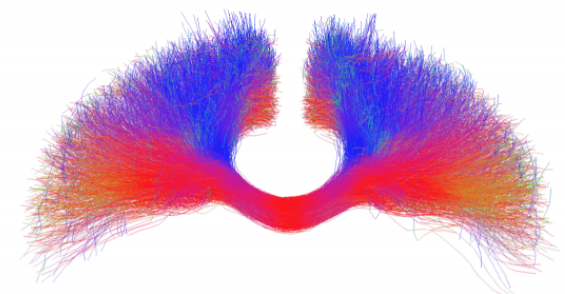

c) Original FOD (native space) b) Orientation priors (native space)

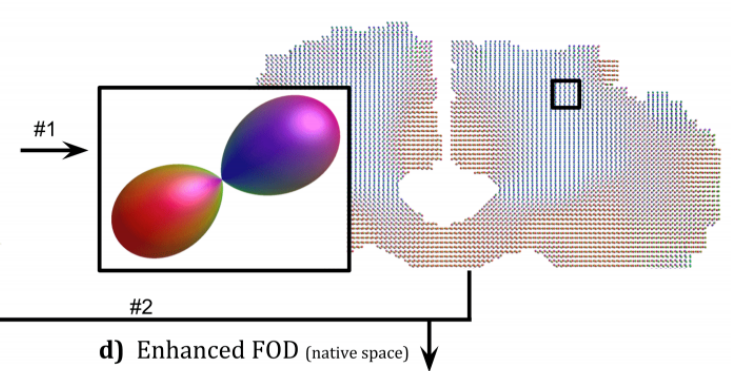

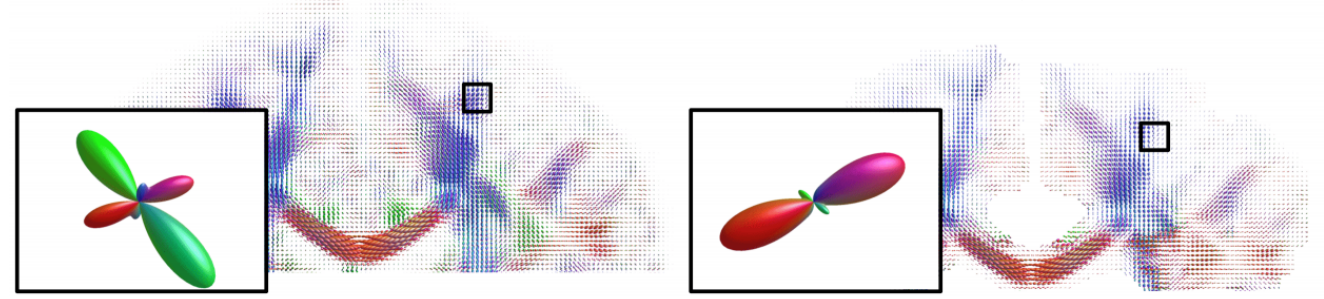

Figure 4: a) Template in a standard space, b) orientation priors in native space, c) initial FOD from CSD, d) pre-computed enhanced FOD.

\section{Building the templates of streamlines}

This initial step is crucial since the template of streamlines affects all additional orientational and anatomical priors used by the BST algorithm. It involves four technical steps (see Figure B.9):

(i) Run a whole-brain or ROI-based tractography technique on the selected subjects.

(ii) Segment the bundles of interest.

(iii) Merge all streamlines of each bundle and each subject in a common space.

(iv) Adjust the density bias of streamlines distribution.

i) Initial tractography. Our approach works in combination with any existing whole-brain streamline-based or iterative tractography algorithm [Jeurissen et al., 2017]. BST will enhance the initial tractography algorithm and retain the properties of the chosen initial algorithm. Global tracking and graph-based/geodesic techniques could also be casted in a BST setting but this is outside the scope of the current paper and will be discussed later.

ii) Segmentation of bundles. Bundles of interest needed for a particular study are usually well-defined in terms of anatomical regions they traverse or connect to [Catani et al., 2002; Catani and De Schotten, 2008]. Moreover, the shape of the bundle is generally agreed upon by various experts [Catani and De Schotten, 2008; Hau et al., 2016] or have often been established from ex vivo Klingler blunt dissections [Fernández-Miranda et al., 2015; Benedictis et al., 2016; Hau et al., 2017]. Hence, this segmentation step of bundles is either done manually or with a semi-automatic filtering or clustering approach. Of 
course, if the number of subjects is large, a manual approach can be tedious and prone to human error. Using ROIs defined by in WM and GM atlases to specify a sequence of rules describing a pathway can be a good alternative [Wassermann et al., 2016]. Another segmentation method is to select or discard streamlines based on shape of cluster using models or anatomical a priori [Voineskos et al., 2009; Guevara et al., 2011; O'donnell et al., 2013; Chekir et al., 2014; Garyfallidis et al., 2017; Zhang et al., 2018].

iii) Bring streamlines to a common space. A common space needs to be chosen. The usual MNI space is a possibility or a tailored template space defined for a specific group of subjects. Each subject is nonlinearly warped to that common space and the deformation field used to move each bundle of interest from each subject into the common space. The deformation involves transforming each point (vertex) of each streamline, using tractmath [Wassermann et al., 2016] or a in-house tool. Once aligned to a common space, bundles of interest are concatenated together into single track file, as seen in Figure 3. The intention of these templates is to represent the general shape and position of bundles.

iv) Correct for the density bias. An individual subject tractogram generally has low density in crossing regions, while extremely dense in regions of one fiber population (easy-to-track parts). The overall shape of a bundle influences this density, i.e. straight bundles will be denser than curved bundles. Since the concept of tract density (streamlines count) in tractography can be a major pitfall that does not accurately represent the underlying WM structure [Jones, 2010; Jbabdi and Johansen-Berg, 2011; Daducci et al., 2016], this non-uniform density bias needs to be removed from the template of streamlines as only the orientation is of interest for our priors. A smart streamline subsampling method based on hierarchical clustering algorithm and minimal direct flip (MDF) distance [Garyfallidis et al., 2012, 2016] is used to overcome this issue. A streamline within $2 \mathrm{~mm}$ of its neighboring streamlines do not carry new information and is removed, without altering the general shape of the template.

As mentioned before, the template of streamlines essentially captures the a priori knowledge of "where" and "how", i.e. where is the bundle originating from, where is going to, and how is it supposed to traverse complex crossing/kissing/branching/fanning regions.

\section{Anatomical priors: Tractography map creation}

The streamlines in the template can now be displaced using the same point-wise method from the template creation (using the inverse deformation field). Once the template of streamlines is in the subject space, a new tracking mask is automatically generated from the voxels intersected by the template streamlines. The streamlines endpoints can also be used to generate a ROI seeding mask. To reduce gyral bias effects and to cover the potential variability across subjects, the seeding and tracking regions are all dilated by $5 \mathrm{~mm}$, as seen in Figure 3 . These masks are used to generate probabilistic exclusion and inclusion maps as well as an interface GM-WM map needed for anatomically-constrained tractography [Smith et al., 2012; Girard et al., 2014]. To prevent streamlines from being generated outside of the white matter, all tracking masks were constrained by the subject's white matter mask. This ensures that our method does not enforce its priors over a subject tissue segmentation, and only the general position within the white matter. 


\section{Orientational priors: Enhancing fiber orientations}

During tractography, streamlines are generated by taking steps using the fiber orientation distribution (FOD) to choose the next direction [Jeurissen et al., 2017]. The value of the FOD is used to weigh the probability of picking a direction. The proposed BST approach uses orientation priors estimated from the template of streamlines to modify the weighting according to the general, or more global, a priori bundle direction. If a streamline is following a specific fiber population, the most well-aligned FOD lobe needs to be prioritized.

To achieve this "prioritization" of FOD lobes, the local orientation histogram from nearby segments of streamlines is computed at every voxel. This method is based on a TOD map estimated from the template of streamlines, as originally introduced [Dhollander et al., 2014]. Improvements related to the FOD field aiming to constraint tractography spatially were proposed in the past [Portegies et al., 2015; Reisert and Kiselev, 2011].

Then, at each voxel, a point-wise multiplication of the FOD and TOD orientation priors is performed, followed by a normalization. As a result, when a lobe is wellaligned with the directions of the priors, the values stay the same. This is observed in Figure 5, where the top row shows TOD maps associated to the pyramidal tracts, the corpus callosum and the arching streamlines respectively. The bottom row shows the corresponding enhanced FOD (E-FOD) associated with each bundle intersecting through the centrum semiovale. Therefore, when a streamline reaches this crossing region, the probability of choosing an appropriate direction is increased. The orientational prior will amplify the desired direction present in the TOD map, and thus improve the directionality of streamlines at the crossing. This concept of using a priori information as well as the observed signal to increase anatomical validity of the decision is borrowed from Bayesian statistics, which is common in neuroimaging [Woolrich et al., 2009; Friston et al., 2002b,a].

If the template of streamlines have crossing or complex fanning, the TOD map will correctly capture this and an appropriate weighting will be achieved. This can be seen in the zoomed picture of Figure 4. On the contrary, lobes that are perpendicular decrease in value as seen in Figure 6, It is important to note that other lobes are not removed, they are simply decreased in amplitude as this information was still present in the diffusion data. As the aim is not to enforced our orientation priors, but simply re-weighting the FOD amplitude, these two scenarios show the impacts in a extreme case and a typical situation.

To ensure that non-existent or erroneous information is not created, a minimal lobevalue threshold is set on the sphere for the orientational priors. This is to ensure that if there is no well-aligned lobe with an amplitude high enough compared to the others, the operation will not increase the values in that direction. A similar threshold was used in Raffelt et al. [2017] to exclude orientations that do not likely represent a fiber population, and used to perform peak extraction on FOD, also to exclude unlikely fiber populations [Tournier et al., 2012; Chamberland et al., 2014; Dell'Acqua et al., 2013]. In our case, we set a relative amplitude threshold to 0.2 (or $20 \%$ ).

\subsection{DWI Datasets}

For this work the templates and experiments were performed with the BIL\&GIN database [Mazoyer et al., 2016]. The datasets are composed of T1-weighted and diffusionweighted images from 39 healthy subjects. The dMRI acquisition consists of 21 gradient 


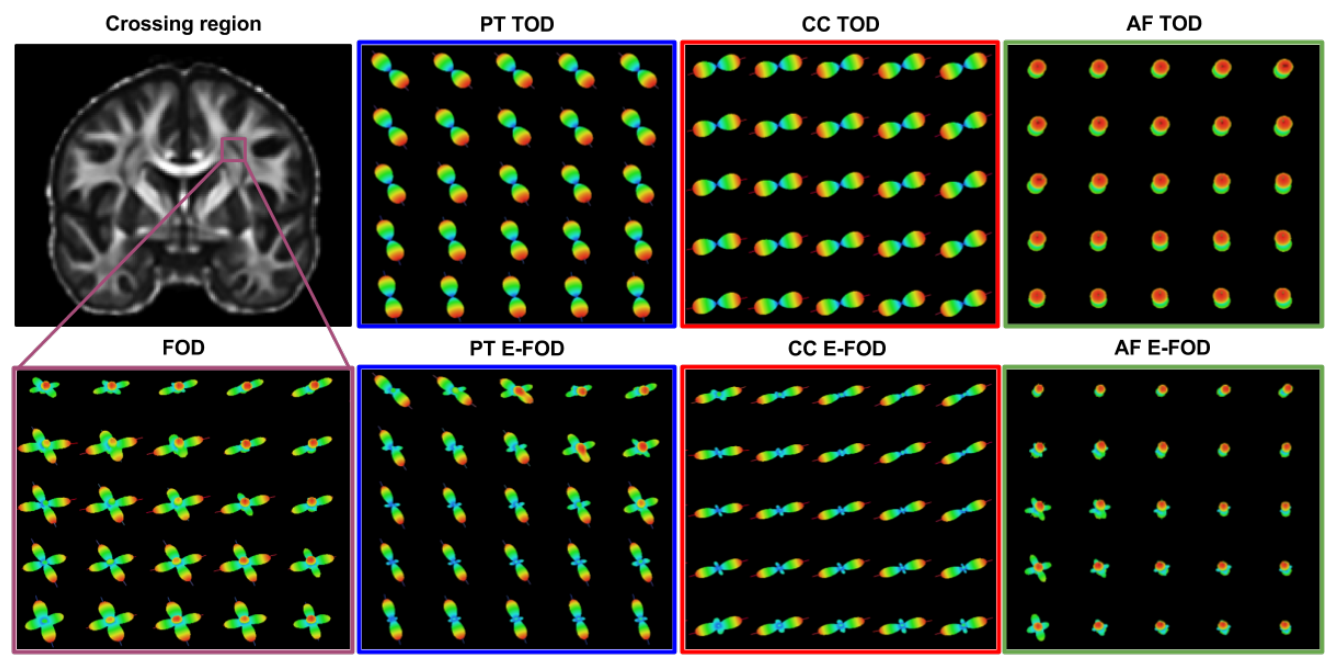

Figure 5: Orientation distribution of the crossing section presented in Figure 1. The first row illustrates the track orientation distribution (TOD) maps generated from the different template (Pyramidal tract, corpus callosum, Arcuate Fasciculus). In the second row, the first FOD vignette presents the original fiber orientation distribution (FOD) in the crossing region followed by enhanced FOD (E-FOD), a combination of the associated template TOD (from the first row) and the original FOD.

a)

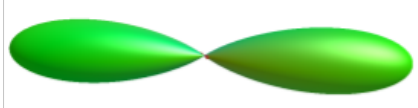

Original FOD

d)

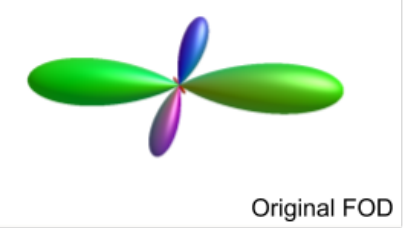

b)

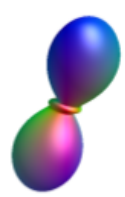

Orientation prior

e)

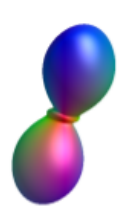

c)

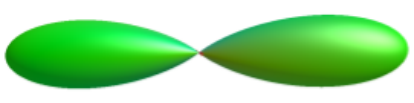

Enhanced FOD

f)

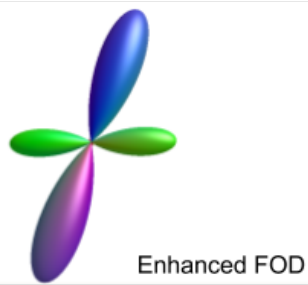

Figure 6: The first row represents a voxel where the FOD (a) does not have any lobe aligned with the orientation prior (b), which results in an enhanced-FOD (c) almost identical to the original. The second row represents a voxel where a FOD (d) with a lobe of sufficient size (above the minimum relative amplitude threshold) globally aligned with the orientation prior (e), which results in an enhanced-FOD (f) with a increase amplitude for that lobe.

directions on half a sphere, the same direction were acquired on the other half of the sphere for averaging, on a single shell $\left(b=1000 \mathrm{~s} / \mathrm{mm}^{2}\right)$ with a $2 \mathrm{~mm}$ isotropic resolution. This scheme was acquired twice for averaging to increase SNR. At each voxel, the FOD was computed using constrained spherical deconvolution (CSD) with spherical harmonics 
of order 6 Tournier et al. [2008]; Descoteaux et al. [2009].

\subsection{Template creation}

For each subject, a whole brain tractogram was reconstructed using the anatomicallyconstrained probabilistic particle filtering tractography (PFT), with recommended default parameters [Girard et al., 2014]. Each tractogram was then segmented using an automatic ROI-based method to extract five bundles of interest, these five bundles will simply be referred to as the left/right pyramidal tract (PT), the corpus callosum (CC) and the left/right arcuate fasciculus (AF). The complete anatomical definitions used by the neuroanatomy expert can be found in the Anatomical Definition Section. We built our own tailored anatomical template using the fractional anisotropy (FA) maps of the 39 subjects with ANTS Multivariate Template Construction script [Avants et al., 2008]. This group average template was used as a common space for the template of streamlines. Subsequently, the 5 bundles of interest, from each subject, were moved into that common space using the nonlinear deformation field from ANTS. Once BOI are concatenated across subject, any redundant streamlines, up to a $2 \mathrm{~mm} \mathrm{MDF}$ distance threshold, are discarded. Moreover, in this work, a leave-one-out approach was used to ensure that the template streamlines generalize to independent datasets. Any experiment using a template performed on a particular subject did not use the subject itself in that template.

\subsection{Experiments, Evaluation, and Statistical analysis}

Probabilistic (Prob) and deterministic (Det), with or without PFT [Girard et al., 2014] were compared, as well as global tractography from MITK Diffusion [Neher et al., 2012]. GM-WM interface seeding was performed, with 5 seeds per voxel, adapted to each bundle of interest (BOI) seeding region (BOI map). Three main sets of parameters were used to compare between classical tracking algorithm and the proposed BST method. To distinguish the impact of improvements from bundle-specific tracking anatomical prior masks and orientational prior enhanced-FOD, three tractography reconstructions were performed:

1. Original FOD with the original exclude/include maps

2. Original FOD with bundle-specific exclude/include maps

3. Enhanced FOD with bundle-specific exclude/include maps

Item 1 corresponds to classical HARDI tracking [Tournier et al., 2012; Jeurissen et al., 2017]. The last item is the full BST method, while item 2 is a point of comparison to help separate the contributions of the anatomical and orientation priors. Finally, we also run global tractography [Kreher et al., 2008; Neher et al., 2012] to test against the proposed BST methods. Detailed descriptions of the experiments are available in the Annexes Section. These lead to five bundles, two tracking evolution approaches (Prob and Det), two algorithm (PFT/no-PFT), and the three compared method (classical, BOI map and BOI \& E-FOD), for a total of 60 tractography files plus one global tractography result per subject. With these 2379 tractograms (61 tractograms times 39 subjects), the automatic bundle segmentation method mentioned earlier (Anatomical Definition) was applied to obtain each bundle. 
Evaluation and statistical analysis were performed using the following measures: bundle volume, weighted-Dice coefficient, average streamlines length, percentage of valid streamlines and computational performance. The volume was obtained by computing a binary mask of every voxel intersected by streamlines, in a $1 \mathrm{~mm}$ isotropic grid [Rheault et al., 2017]. The weighted-Dice was introduced by Cousineau et al. [2017] to obtain a value representing spatial agreement that takes account streamlines density. Using the Dice coefficient as a spatial agreement metric will be severely penalized for spurious streamlines. The overall shape of bundles of interests were expected to be similar across subjects. The strict anatomical definitions lead to comparable bundles across subjects once registered in a common space, especially for major WM pathways. In addition, an intra-subject approach would not have provided direct information regarding the quality of a bundle reconstruction. Lack of fanning reaching the cortex could be reproducible and the spatial agreement high without showing improvement compared to the expected shape. Since the bundles of interests in this project (AF, CC, PT) are major WM pathways, they are generally anatomically comparable across subjects, i.e. the spatial agreement is expected to be high, without necessarily reaching a perfect score.

The percentage of valid streamlines is the proportion of generated streamlines respecting the anatomical definition of a bundle from the expert (see Anatomical Definition Section) and a length threshold compared to the total amount of streamlines that were originally generated. All outputs from tractography have to respect the algorithm constraints (tracking mask, angular threshold, spherical function threshold), however the length threshold was applied only for bundles. segmentation. In the context of our experiments, the notion of valid and invalid streamlines vary with the bundle of interest investigated. A valid streamline for a particular BOI will be considered invalid for all other BOI. For example, a BOI interface of 100 voxels with 5 seeds per voxel would launch 500 streamlines, if 25 streamlines are segmented (considered valid), this would amount to $5 \%$ of valid streamlines. Computational performance (efficiency), represents the total number of tractography iterations, i.e. tracking steps, leading to valid streamline points. This is slightly different than the previous percentage of valid streamlines measure, especially with the BOI tracking mask, because most invalid streamlines end up having less than 50 steps while valid streamlines end up with more 500 steps. This metric is better than streamlines count to represent the improvement in terms of computational performance.

For statistical analysis, all subjects and bundles were analyzed separately. Then, to highlight general trends, results were averaged. The analysis was performed with all streamlines registered in the FA template space. This alignment, allowed to compute overlaps between subjects and to normalize the values obtained for the volume and average length. This procedure ensures that variations in volume or length does not come from disparities in brain size of the subject but rather from the tracking itself.

\section{Results}

To simplify reading of tables, graphs and figures, the focus will be on probabilistic tracking with particle filter tractography (PFT), but all evaluation data, graphs and figures are available in the Annexes Section. Observations and trends are similar and as expected. Moreover, MITK Global tractography was also benchmarked on the same measures to ensure an adequate comparison to state-of-the-art tractography algorithms. 
Figure 7 shows segmentations of PT, CC, and AF bundles of interest with a probabilistic PFT algorithm, comparing the use of original FOD and PFT maps to the proposed BST approach using the enhanced FOD with the modified PFT maps. The reconstructed bundles of interest from the classical tracking show some degree of fanning, but the number of streamlines generated was not sufficient to fully recover the spatial extent of bundles. All bundles reconstructed using the masking priors and the enhanced FOD show an increase in both bundle coverage and quality of the fanning. For the PT, the lateral portions of the pre- and post-central gyri are better covered while they are almost non-existent with standard PFT. Similarly, the CC has the fanning fully covering the pre-central and post-central gyri. As for the AF, the spatial extent in the frontal and temporal lobes is increased, but streamlines are also reaching more laterally into the gyri. The quantitative impact of the improved fanning directly impacted the volumes of the reconstructed bundles, as seen in the first column of Table 1.

Figure 8 shows the trends for each measures of the analysis, where each graph represents the changes of all bundles across the three sets of tractography parameters. The impacts are not the same for each bundle, as the shape, size and the underlying anatomy are inherently different. Error bars in the histograms represent the average values and their standard deviation across 39 subjects. As the histograms can be hard to compare, values are also shown in Table 1.

In Figure 8 a), an increase in volume for all bundles is measured when the priors
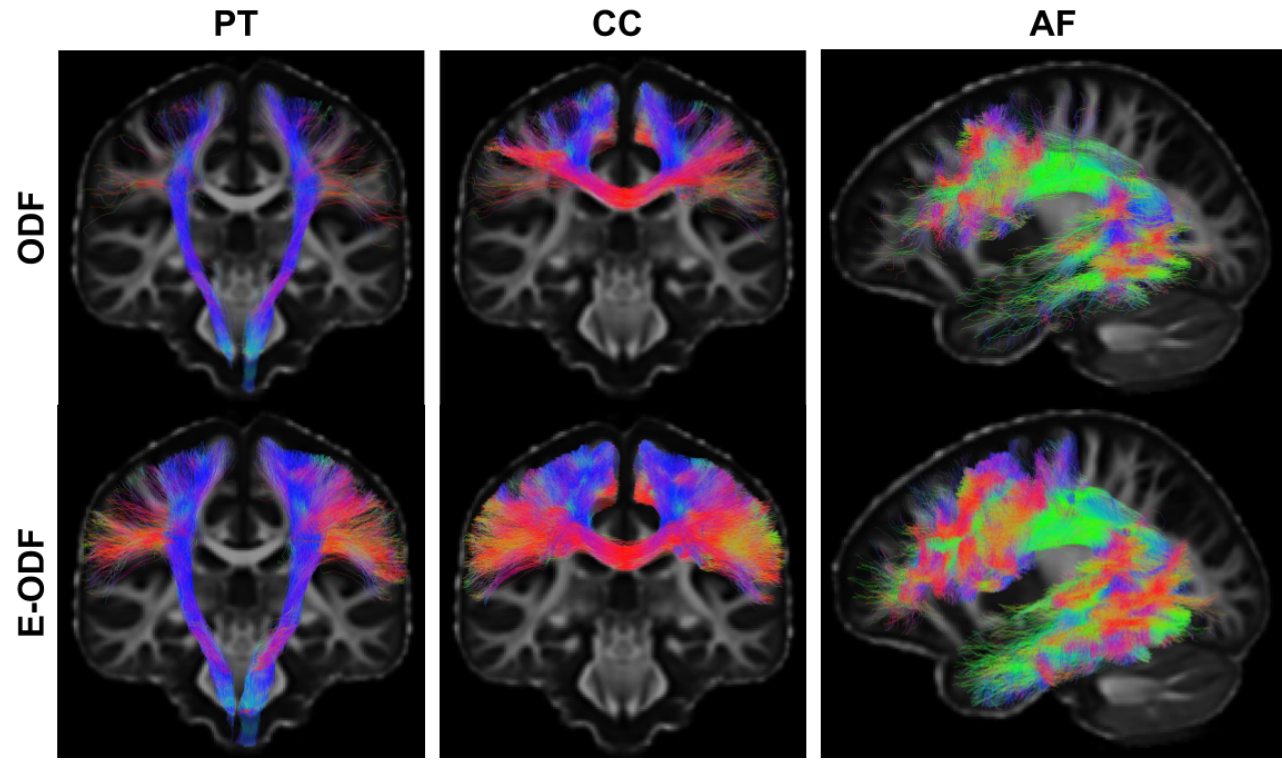

Figure 7: Examples of resulting PFT and BST algorithms for a single subject. The first row shows results from standard tracking of the $\mathrm{PT}, \mathrm{CC}$ and $\mathrm{AF}$, while the second row shows results from the proposed bundle-specific tracking. Of note, at no point tracking is allowed outside of the initial white matter tracking mask. It involves that no streamline travel in gray matter or CSF and the impression of going over the sulci which can be observed in the second row is due to coronal view lacking perspective. 
Left Pyramidal Tract

\begin{tabular}{|l|l|l|c|c|c|c|c|}
\hline & $\begin{array}{l}\text { Local } \\
\text { model }\end{array}$ & Mask & $\begin{array}{c}\text { Bundle } \\
\text { vol. }\left(\mathrm{cm}^{3}\right)\end{array}$ & Dice index & $\begin{array}{c}\text { Average } \\
\text { length }(\mathrm{cm})\end{array}$ & $\begin{array}{c}\text { \% of valid } \\
\text { streamlines }\end{array}$ & $\begin{array}{c}\text { Efficiency } \\
(\%)\end{array}$ \\
\hline 1. & FOD & WM & $9.80 \pm 3.42$ & $0.63 \pm 0.12$ & $12.58 \pm 0.38$ & $0.29 \pm 0.20$ & $1.30 \pm 0.92$ \\
\hline 2. & FOD & BOI & $14.70 \pm 4.00$ & $0.71 \pm 0.10$ & $12.66 \pm 0.32$ & $0.67 \pm 0.38$ & $4.01 \pm 2.45$ \\
\hline 3. & E-FOD & BOI & $\mathbf{2 7 . 9 5} \pm \mathbf{6 . 2 4}$ & $\mathbf{0 . 8 6} \pm \mathbf{0 . 0 6}$ & $12.16 \pm 0.30$ & $\mathbf{3 . 9 2} \pm \mathbf{1 . 9 5}$ & $\mathbf{1 1 . 7 3} \pm \mathbf{6 . 4 9}$ \\
\hline
\end{tabular}

Right Pyramidal Tract

\begin{tabular}{|l|l|l|c|c|c|c|c|}
\hline & $\begin{array}{l}\text { Local } \\
\text { model }\end{array}$ & Mask & $\begin{array}{c}\text { Bundle } \\
\text { vol. }\left(\mathrm{cm}^{3}\right)\end{array}$ & Dice index & $\begin{array}{c}\text { Average } \\
\text { length }(\mathrm{cm})\end{array}$ & $\begin{array}{c}\text { \% of valid } \\
\text { streamlines }\end{array}$ & $\begin{array}{c}\text { Efficiency } \\
(\%)\end{array}$ \\
\hline 1. & FOD & WM & $6.56 \pm 2.74$ & $0.51 \pm 0.15$ & $12.03 \pm 0.32$ & $0.14 \pm 0.10$ & $0.58 \pm 0.42$ \\
\hline 2. & FOD & BOI & $11.08 \pm 3.42$ & $0.64 \pm 0.13$ & $12.07 \pm 0.26$ & $0.38 \pm 0.22$ & $2.21 \pm 1.32$ \\
\hline 3. & E-FOD & BOI & $\mathbf{2 5 . 2 7} \pm \mathbf{5 . 3 3}$ & $\mathbf{0 . 8 3} \pm \mathbf{0 . 0 6}$ & $11.59 \pm 0.24$ & $\mathbf{2 . 4 2} \pm \mathbf{1 . 1 9}$ & $\mathbf{7 . 2 6} \pm \mathbf{3 . 9 5}$ \\
\hline
\end{tabular}

Corpus Callosum

\begin{tabular}{|l|l|l|c|c|c|c|c|}
\hline & $\begin{array}{l}\text { Local } \\
\text { model }\end{array}$ & Mask & $\begin{array}{c}\text { Bundle } \\
\text { vol. }\left(\mathrm{cm}^{3}\right)\end{array}$ & Dice index & $\begin{array}{c}\text { Average } \\
\text { length }(\mathrm{cm})\end{array}$ & $\begin{array}{c}\text { \% of valid } \\
\text { streamlines }\end{array}$ & $\begin{array}{c}\text { Efficiency } \\
(\%)\end{array}$ \\
\hline 1. & FOD & WM & $25.78 \pm 6.58$ & $0.65 \pm 0.11$ & $11.25 \pm 0.37$ & $0.72 \pm 0.33$ & $2.89 \pm 1.39$ \\
\hline 2. & FOD & BOI & $49.51 \pm 7.40$ & $0.78 \pm 0.07$ & $11.49 \pm 0.31$ & $2.68 \pm 0.85$ & $14.09 \pm 5.17$ \\
\hline 3. & E-FOD & BOI & $\mathbf{8 7 . 2 3} \pm \mathbf{7 . 7 1}$ & $\mathbf{0 . 8 6} \pm \mathbf{0 . 0 6}$ & $11.53 \pm 0.26$ & $\mathbf{1 2 . 2 0} \pm \mathbf{3 . 4 6}$ & $\mathbf{3 0 . 3 2} \pm \mathbf{9 . 9 6}$ \\
\hline
\end{tabular}

Left Arcuate Fasciculus

\begin{tabular}{|l|l|l|c|c|c|c|c|}
\hline & $\begin{array}{l}\text { Local } \\
\text { model }\end{array}$ & Mask & $\begin{array}{c}\text { Bundle } \\
\text { vol. }\left(\mathrm{cm}^{3}\right)\end{array}$ & Dice index & $\begin{array}{c}\text { Average } \\
\text { length }(\mathrm{cm})\end{array}$ & $\begin{array}{c}\text { \% of valid } \\
\text { streamlines }\end{array}$ & $\begin{array}{c}\text { Efficiency } \\
(\%)\end{array}$ \\
\hline 1. & FOD & WM & $54.21 \pm 9.49$ & $0.70 \pm 0.08$ & $10.61 \pm 1.30$ & $1.23 \pm 0.45$ & $4.92 \pm 1.94$ \\
\hline 2. & FOD & BOI & $56.27 \pm 10.06$ & $0.70 \pm 0.08$ & $10.78 \pm 1.26$ & $1.48 \pm 0.55$ & $7.99 \pm 3.28$ \\
\hline 3. & E-FOD & BOI & $\mathbf{1 1 0 . 0 3} \pm \mathbf{1 0 . 2 3}$ & $\mathbf{0 . 7 5} \pm \mathbf{0 . 0 6}$ & $\mathbf{1 2 . 2 9} \pm \mathbf{0 . 7 2}$ & $\mathbf{9 . 7 4} \pm \mathbf{2 . 8 0}$ & $\mathbf{3 2 . 2 6} \pm \mathbf{1 1 . 1 4}$ \\
\hline
\end{tabular}

Right Arcuate Fasciculus

Right Arcuate Fasciculus
\begin{tabular}{|l|l|l|c|c|c|c|c|}
\hline $\begin{array}{l}\text { Local } \\
\text { model }\end{array}$ & Mask & $\begin{array}{c}\text { Bundle } \\
\text { vol. }\left(\mathrm{cm}^{3}\right)\end{array}$ & Dice index & $\begin{array}{c}\text { Average } \\
\text { length }(\mathrm{cm})\end{array}$ & $\begin{array}{c}\% \text { of valid } \\
\text { streamlines }\end{array}$ & $\begin{array}{c}\text { Efficiency } \\
(\%)\end{array}$ \\
\hline 1. & FOD & WM & $35.89 \pm 9.97$ & $0.58 \pm 0.13$ & $9.35 \pm 2.44$ & $0.77 \pm 0.36$ & $2.71 \pm 1.60$ \\
\hline 2. & FOD & BOI & $37.99 \pm 10.26$ & $0.59 \pm 0.13$ & $9.55 \pm 2.37$ & $0.95 \pm 0.44$ & $4.62 \pm 2.69$ \\
\hline 3. & E-FOD & BOI & $\mathbf{8 1 . 8 4} \pm \mathbf{1 2 . 9 9}$ & $\mathbf{0 . 8 0} \pm \mathbf{0 . 0 6}$ & $\mathbf{1 1 . 6 0} \pm \mathbf{0 . 8 2}$ & $\mathbf{5 . 9 5} \pm \mathbf{2 . 4 6}$ & $\mathbf{1 8 . 8 9} \pm \mathbf{9 . 1 5}$ \\
\hline
\end{tabular}

Table 1: Quantitative results are shown separately for each bundle of interest from three experiments, measures of the 39 validation subjects were averaged together. Values for average and standard deviation are formatted to simplify interpretation. All measures, except average length, are improved by our approach when compared to classical tractography (approach 1 and 2), the difference is statistically significant $(\mathrm{p}<0.01)$. Average length remains stable across method, however it was significantly higher for both arcuate fasciculus.

are used. The AF are the only bundles with no significant increase between the tracking using no a priori masking and the tracking using only the a priori masking, however the volume significantly increases when enhanced FOD are used. The standard deviation, for methods 2 and 3 , in proportion to the average, is decreasing. The volume of the reconstructed PT using method 3 is approximately $400 \%$ of the volume obtained from classical tracking, $300 \%$ for the $\mathrm{CC}$ and $200 \%$ for the $\mathrm{AF}$.

The reproducibility across datasets was measured by the Weighted-Dice coefficient. Tractography without priors poorly performed with values between 0.5 (PT right) and 0.7 (AF left). In Figure 8 b), an increase in overlap for all BOI is observed when E-FOD are used, with Weigthed-Dice values all more or less 0.8. Similarly to the volume, the $\mathrm{AF}$ are the only bundles with no significant increase between methods 1 and 2 , while 
the overlap significantly increases when enhanced FOD are used. For methods 2 and 3 , the standard deviation of the Weighted-Dice coefficient is decreasing as the average coefficient is increasing. For all bundles, except the left AF, the Weigthed-Dice increases by $20 \%$ when method 3 is used instead of the classical tracking.

The average length and standard deviation was computed to evaluate the shape
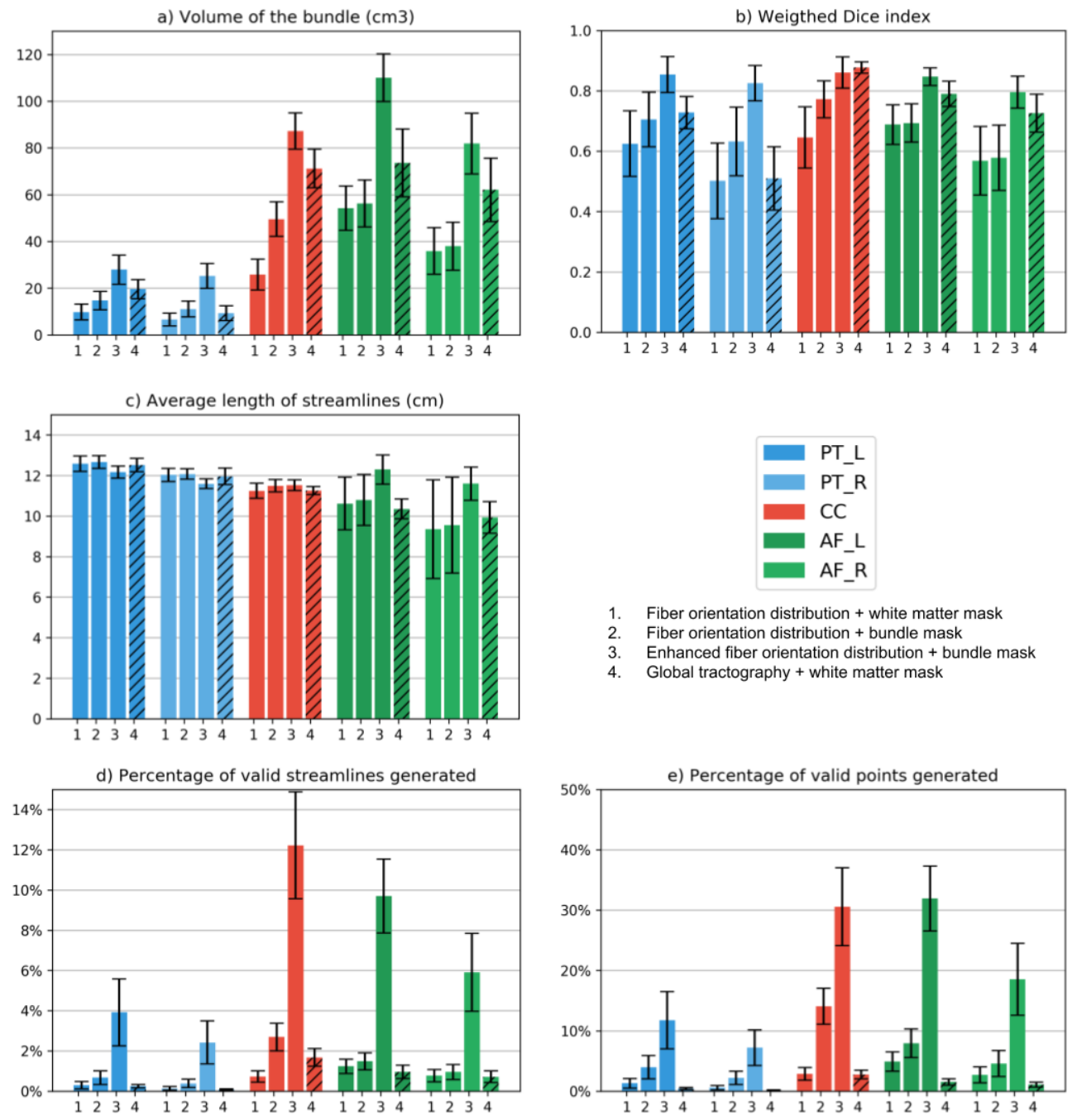

Figure 8: Quantitative measures for each BOI across the 3 tracking algorithms. Results from global tractography is added to provide more context, but comparaison is partly "unfair" as many variables cannot be controlled in the same way. The Results are shown for probabilistic tracking with PFT. Graphs for deterministic and probabilistic versions of the algorithms with and without PFT are also available in the Annexes Section 
coherence of each bundle. Globally, the average length of most BOI remained similar for each method, as seen in Figure $8 \mathrm{c}$ ). However, for the AF, going from methods 2 to 3 , the average length increased and the standard deviation decreased. This can be observed more easily in Table 1. As mentioned earlier (Figure 7), this is due to streamlines reaching further inside the lateral gyri.

Finally Figure 8 d) and e) and the last two columns in Table 1 show measures of efficiency. The use of adapted tracking masks (method 2) is not remarkably influencing the percentage of valid streamlines. However, integrating enhanced FOD (method 3) significantly increases efficiency. A tractogram with one million streamlines, each streamline with more or less one hundred points, would need one hundred million iterations. However, not all streamlines have the same number of points depending on their length. When adapted tracking masks (method 2) are used, most invalid streamlines are quickly stopped and thus a large number of iterations avoided. Also, it is important to note that the percentage of valid streamlines and efficiency are improved but that they are still under $15 \%$ and $40 \%$ respectively, which leaves plenty of room for further improvements.

Similar trends can be observed in the Annexes Section for probabilistic tractography (without particle filtering). Bundle-specific tractography also improves reconstruction when using deterministic algorithms, the difference between classical deterministic and deterministic bundle-specific tractography was statistically significant $(\mathrm{p}<0.01)$. This shows that most algorithms using FOD as an input need orientational priors to better reconstruct bundle of interest.

Results from MITK global tractography [Neher et al., 2012] were overall superior to all classic tractography algorithms used for the experiments, as seen in Annexes Figures B.10,B.11,B.12,B.13. However, bundle-specific probabilistic tractography always generate bundles with higher volume and higher reproducibility across subjects compared to global tractography. The orientational prior used with probabilistic tractography (with or without PFT) achieve better results while decreasing computational needs.

\section{Discussion}

Our bundle-specific tractography algorithm is designed to address the poor-spatialextent challenge and the sensitivity-specificity trade-off of tractography. Contributions are: i) novel bundle-specific anatomical and orientational priors directly incorporated in the tractography process to boost streamline tracking in hard-to-track regions, ii) results that show increased reproducibility, sensitivity, and specificity of PT, CC, AF bundle reconstructions in the complex centrum semiovale region, and iii) a new BST algorithm with increased efficiency and reduced overall computation time. The main methodological innovation is the novel local fiber orientation distribution enhancement methodology based on white matter bundle priors. These additions were shown to yield better spatial coverage of the pyramidal tract, corpus callosum, and arcuate fasciculus, increasing the quality of the extent of fanning reaching the cortex, helping to accurately represent the shape of each fascicle. Increase in volume is not a direct measure of the quality of a bundle reconstruction. The use of a strict anatomical definition based on the literature is fundamental to correctly address this concern. The definition was designed to reduce as much as possible the number of false positives, meaning that significant 
increase in volume cannot be accounted by an increase in outliers, spurious streamlines or contributions from other bundles. As mentioned earlier, tracking is never allowed outside of the initial white matter tracking mask. Hence, increase in volume cannot come from "new voxels" added by our algorithm. The increase in volume was observed in regions that initially lack fanning reaching the cortex, which is a common difficulty for classical tractography [Girard et al., 2014; St-Onge et al., 2017]. It is important to note that the FOD enhancement affects the mathematical properties of the FOD. The E-FOD should not be used to compute FOD metrics such as the AFD [Raffelt et al., 2017] or even NuFO [Tournier et al., 2008] since lobes amplitude changed and no longer agree with the DWI signal.

\section{Template creation needed for Bundle-Specific Tractography}

The creation of an appropriate template of streamlines is crucial for the proposed method, as mentioned in the Methods Section. The input bundle of interest of the template needs to be strictly segmented and cleaned of spurious streamlines, while still representing all the potential anatomical variability across subjects. We acknowledge that anatomical definition of WM bundles can vary among experts and that there exist several open controversies in the WM neuroanatomy literature [Forkel et al., 2014; Meola et al., 2015; Hau et al., 2017]. However, having a consensus on the definition of the bundle is not necessary to showcase the potential of our Bundle-Specific Tractography. Neuroanatomists can debate and converge on the precise definitions of anatomical bundles, which can then be adjusted into our BST method.

Moreover, the nonlinear deformation of the template to the subject's native space is done under the assumption that ANTS performs an adequate registration in-between FA maps. In the case of a different study, with pathology or large brain alterations, such as a tumor, cyst, severe atrophy, or missing brain regions, the quality of the alignment would need to be guaranteed manually. Using a tailored template based on a specific cohort enables our proposed BST method to be fully automatic without relying on manually placed ROIs to enforce certain directions and ending regions. Pathological brains already raise numerous challenges for tissue segmentation, registration, classical tractography and analysis, which all require case-by-case decisions and tuning. For example, WM lesions for Alzheimer's disease, multiple sclerosis, traumatic brain injuries do not have the same underlying causes and tractography could sometimes be allowed to go through them or not. When all conditions are met and potential pitfalls related to the pathology are accounted for, BST can yield interesting results and can have positive impacts for challenging datasets, as illustrated on the fornix reconstruction in an aging study [Rheault et al., 2018].

As atlases of streamlines become more widely available, such as the one of Yeh and Tseng [2011]; Wasserthal et al. [2018a], different research groups will be able to use these without having to build their own. As an attempt to facilitate its use and guarantee the quality of the anatomical priors for well-known pathways, a WM atlas dedicated to the BST has been designed. This is a step in the right direction to include anatomical priors to improve tractography and address some of the challenges raised in Maier-Hein et al. [2017]. To facilitate future investigations and help other researchers to use our method, a Nextflow pipeline [Di Tommaso et al., 2017] has been developed. By providing configuration files, a lightweight WM atlases of bundles and a singularity environment [Kurtzer et al., 2017], this pipeline will simplify the usage of our method. Also, researchers 
with specific needs can easily customize the pipeline and the configuration file and obtain exactly what they need.

\section{Bundle volume and spatial coverage}

The priors on the regions of interests and local orientations noticeably improve the virtual dissection results by increasing the total volume and the fanning extent. FOD enhancement helps to overcome tracking difficulties in the crossing regions, allowing more streamlines to respect the anatomical definition of the bundle of interest (see Anatomical Definition Section). Difficulties encountered in the crossing region would have required a higher number of seeds per voxel to obtain an appropriate reconstruction for the classical method, however this approach does not ensure to fully capture the spatial extent. When FODs are enhanced to support the main directionality of a bundle of interest, crossing regions become less disorienting during the tractography, thus increasing the number of streamlines to be considered during segmentation. All this while being more efficient, in term of valid streamlines after segmentation.

As the fanning component of the bundles represent the majority of their volume, improved fanning has a quantitative impact on the volume. For example, the fanning of the PT and CC occurs in the 3-way crossing region (Figure 1), traditionally resulting in a small preponderance of streamlines reaching lateral portions of the primary motor and somatosensory cortex. The priors largely impact the volume of the bundles (first column of Table 1). The use of a priori seeding tracking masks increases volume as particle filtering tractography enforces streamline termination in GM regions. The usage of enhanced FOD increases the volume even more by reducing the potential confusion in crossings, as picking a bad direction at each step quickly accumulates in terms of error.

\section{Reproducibility}

Bundles of interest considered in this project are known to have anatomical variability at the micro-scale, but at the macro-scale (on the whole bundle level) the AF, CC and PT are expected to cover the same regions (once in a common space) after the segmentation described in the Anatomical Definition Section. We acknowledge that an identical coverage is not expected, meaning the weighted-dice should not be expected to be 1.0. However, poor spatial agreement (inter-subject) with the classical tractography method mainly comes from poor fanning, a typical solution to this problem is to seed more until the expected shape is obtained and fanning is adequate. The low weighteddice value seen from classical method is influenced by the high variability of the fanning (CC, PT) or from the early-stop of streamlines in the frontal and temporal lobe (AF). The goal was not to obtain a value of 1.0. The goal was to reduce the variability in these regions as they were caused by tractography limitations and not explained by the underlying anatomy. Intra-subject reproducibility could have hidden this pitfall as a lack of fanning could be reproducible (intra-subject) and would not have reflected if the expected anatomical shape was achieved.

\section{Computational Performance}

Immediately stopping any streamline getting out of the bundle of interest region using a priori imposed dilated masks directly reduces the number of tractography iterations and saves computation time without altering the resulting dissection. Such stopping criteria also decreases the rate of false positives, as streamlines must follow a reasonable 
path to be considered valid. The efficiency of BST is also increased since streamlines follow the main direction of the BOI instead of leaving prematurely the tracking mask without reaching ROI endpoints. By following the appropriate direction in the crossing, the streamlines have more chance to respect all the bundle definitions, resulting in a higher amount of valid streamlines.

Performance is not just about number of streamlines. The increase in performance combined with the increase in volume and overlap between subjects (Table 1) shows that using the proposed method helps to reach stable results more rapidly, while improving the quality of the bundle of interest.

\section{Future Work}

Since the output of the proposed method is in the form of FOD in a widespread format (spherical harmonics) and binary masks, any algorithm that accepts such input could be easily adapted. Other forms of tracking (Dipy [Garyfallidis et al., 2014], FSL [Behrens et al., 2007], MRtrix [Tournier et al., 2012], Real-time tractography [Chamberland et al., 2014], even Global tractography [Kreher et al., 2008; Christiaens et al., 2015; Reisert et al., 2011]) could be tried. Algorithms designed to add more a priori could also be tried alongside enhanced-FOD such as surface-enhanced tractography [St-Onge et al., 2017] and microstructure-informed tractography (AxTract) [Girard et al., 2015]).

\section{Conclusion}

We developed a new bundle-specific tracking (BST) algorithm incorporating novel anatomical orientational priors directly into the streamline tracing process to address the poor-spatial-extent challenge and sensitivity-specificity trade-off of WM bundle reconstruction using tractography. This fully automatic method exploits information extracted from a template of streamlines of bundles of interest to enhance the local modeling in the desired direction using the a priori fiber orientation distribution. It was shown that the proposed approach improves the spatial coverage and increases the quality of the fanning in crossing regions, while reducing computational need. Since a stable volume and sufficient anatomically valid streamlines are reconstructed faster than with classical techniques, this method helps the exploration of structural connectivity with more confidence and less biases in white matter bundles metrics. This could have a positive impact on the neurosciences community using dMRI tractography tract-based and connectivity-based analyses.

\section{Acknowledgements}

Thank you to Philippe Poulin and Jasmeen Sidhu for their insights which helped shape this paper. A special thank you to the funding sources for this work, the FRQNT and CREATE-MIA programs. Thank you to the Neuroinformatics Chair of the Sherbrooke University which helped push forward neurosciences research.

\section{References}

Avants, B.B., Epstein, C.L., Grossman, M., Gee, J.C., 2008. Symmetric diffeomorphic image registration with cross-correlation: evaluating automated labeling of elderly and neurodegenerative brain. Medical image analysis $12,26-41$. 
Basser, P.J., Pajevic, S., Pierpaoli, C., Duda, J., Aldroubi, A., 2000. In vivo fiber tractography using dt-mri data. Magnetic resonance in medicine 44, 625-632.

Behrens, T.E., Berg, H.J., Jbabdi, S., Rushworth, M.F., Woolrich, M.W., 2007. Probabilistic diffusion tractography with multiple fibre orientations: What can we gain? Neuroimage 34, 144-155.

Benedictis, A., Petit, L., Descoteaux, M., Marras, C.E., Barbareschi, M., Corsini, F., Dallabona, M., Chioffi, F., Sarubbo, S., 2016. New insights in the homotopic and heterotopic connectivity of the frontal portion of the human corpus callosum revealed by microdissection and diffusion tractography. Human brain mapping 37, 4718-4735.

Calamante, F., Smith, R.E., Tournier, J.D., Raffelt, D., Connelly, A., 2015. Quantification of voxel-wise total fibre density: investigating the problems associated with track-count mapping. Neuroimage 117, $284-293$.

Catani, M., Allin, M.P., Husain, M., Pugliese, L., Mesulam, M.M., Murray, R.M., Jones, D.K., 2007. Symmetries in human brain language pathways correlate with verbal recall. Proceedings of the National Academy of Sciences 104, 17163-17168.

Catani, M., De Schotten, M.T., 2008. A diffusion tensor imaging tractography atlas for virtual in vivo dissections. cortex 44, 1105-1132.

Catani, M., Howard, R.J., Pajevic, S., Jones, D.K., 2002. Virtual in vivo interactive dissection of white matter fasciculi in the human brain. Neuroimage 17, 77-94.

Chamberland, M., Scherrer, B., Prabhu, S.P., Madsen, J., Fortin, D., Whittingstall, K., Descoteaux, M., Warfield, S.K., 2017. Active delineation of meyer's loop using oriented priors through magnetic tractography (magnet). Human brain mapping 38, 509-527.

Chamberland, M., Whittingstall, K., Fortin, D., Mathieu, D., Descoteaux, M., 2014. Real-time multipeak tractography for instantaneous connectivity display. Frontiers in neuroinformatics 8, 59.

Chekir, A., Descoteaux, M., Garyfallidis, E., Côté, M.A., Boumghar, F.O., 2014. A hybrid approach for optimal automatic segmentation of white matter tracts in hardi, in: Biomedical Engineering and Sciences (IECBES), 2014 IEEE Conference on, IEEE. pp. 177-180.

Chenot, Q., Tzourio-Mazoyer, N., Rheault, F., Descoteaux, M., Crivello, F., Zago, L., Mellet, E., Jobard, G., Joliot, M., Mazoyer, B., et al., 2018. A probabilistic atlas of the human pyramidal tract in 410 healthy participants. bioRxiv , 251108.

Christiaens, D., Reisert, M., Dhollander, T., Sunaert, S., Suetens, P., Maes, F., 2015. Global tractography of multi-shell diffusion-weighted imaging data using a multi-tissue model. Neuroimage 123, 89-101.

Côté, M.A., Girard, G., Boré, A., Garyfallidis, E., Houde, J.C., Descoteaux, M., 2013. Tractometer: towards validation of tractography pipelines. Medical image analysis 17, 844-857.

Cousineau, M., Jodoin, P.M., Garyfallidis, E., Côté, M.A., Morency, F.C., Rozanski, V., GrandMaison, M., Bedell, B.J., Descoteaux, M., 2017. A test-retest study on parkinson's ppmi dataset yields statistically significant white matter fascicles. NeuroImage: Clinical 16, 222-233.

Daducci, A., Dal Palú, A., Descoteaux, M., Thiran, J.P., 2016. Microstructure informed tractography: pitfalls and open challenges. Frontiers in neuroscience 10, 247.

Daducci, A., Dal Palù, A., Lemkaddem, A., Thiran, J.P., 2015. Commit: convex optimization modeling for microstructure informed tractography. IEEE transactions on medical imaging 34, $246-257$.

Dayan, M., Monohan, E., Pandya, S., Kuceyeski, A., Nguyen, T.D., Raj, A., Gauthier, S.A., 2016. Profilometry: a new statistical framework for the characterization of white matter pathways, with application to multiple sclerosis. Human brain mapping 37, 989-1004

Dell'Acqua, F., Simmons, A., Williams, S.C., Catani, M., 2013. Can spherical deconvolution provide more information than fiber orientations? hindrance modulated orientational anisotropy, a true-tract specific index to characterize white matter diffusion. Human brain mapping 34, 2464-2483.

Descoteaux, M., Deriche, R., Knosche, T.R., Anwander, A., 2009. Deterministic and probabilistic tractography based on complex fibre orientation distributions. IEEE transactions on medical imaging 28, 269-286.

Desikan, R.S., Ségonne, F., Fischl, B., Quinn, B.T., Dickerson, B.C., Blacker, D., Buckner, R.L., Dale, A.M., Maguire, R.P., Hyman, B.T., et al., 2006. An automated labeling system for subdividing the human cerebral cortex on mri scans into gyral based regions of interest. Neuroimage 31, 968-980.

Dhollander, T., Emsell, L., Van Hecke, W., Maes, F., Sunaert, S., Suetens, P., 2014. Track orientation density imaging (todi) and track orientation distribution (tod) based tractography. NeuroImage 94, $312-336$.

Di Tommaso, P., Chatzou, M., Floden, E.W., Barja, P.P., Palumbo, E., Notredame, C., 2017. Nextflow enables reproducible computational workflows. Nature biotechnology 35, 316-319.

Fernández-Miranda, J.C., Wang, Y., Pathak, S., Stefaneau, L., Verstynen, T., Yeh, F.C., 2015. Asym- 
metry, connectivity, and segmentation of the arcuate fascicle in the human brain. Brain Structure and Function 220, 1665-1680

Forkel, S.J., de Schotten, M.T., Kawadler, J.M., Dell'Acqua, F., Danek, A., Catani, M., 2014. The anatomy of fronto-occipital connections from early blunt dissections to contemporary tractography. Cortex 56, 73-84.

Friston, K.J., Glaser, D.E., Henson, R.N., Kiebel, S., Phillips, C., Ashburner, J., 2002a. Classical and bayesian inference in neuroimaging: applications. Neuroimage $16,484-512$.

Friston, K.J., Penny, W., Phillips, C., Kiebel, S., Hinton, G., Ashburner, J., 2002b. Classical and bayesian inference in neuroimaging: theory. NeuroImage 16, 465-483.

Garyfallidis, E., Brett, M., Amirbekian, B., Rokem, A., Van Der Walt, S., Descoteaux, M., NimmoSmith, I., 2014. Dipy, a library for the analysis of diffusion mri data. Frontiers in neuroinformatics 8 , 8.

Garyfallidis, E., Brett, M., Correia, M.M., Williams, G.B., Nimmo-Smith, I., 2012. Quickbundles, a method for tractography simplification. Frontiers in neuroscience 6, 175.

Garyfallidis, E., Côté, M.A., Rheault, F., Descoteaux, M., 2016. Quickbundlesx: sequential clustering of millions of streamlines in multiple levels of detail at record execution time. ISMRM2016 (Singapore)

Garyfallidis, E., Côté, M.A., Rheault, F., Sidhu, J., Hau, J., Petit, L., Fortin, D., Cunanne, S., Descoteaux, M., 2017. Recognition of white matter bundles using local and global streamline-based registration and clustering. NeuroImage .

Ghaziri, J., Tucholka, A., Girard, G., Houde, J.C., Boucher, O., Gilbert, G., Descoteaux, M., Lippé, S., Rainville, P., Nguyen, D.K., 2015. The corticocortical structural connectivity of the human insula. Cerebral cortex 27, 1216-1228.

Girard, G., Fick, R., Descoteaux, M., Deriche, R., Wassermann, D., 2015. Axtract: microstructure-driven tractography based on the ensemble average propagator, in: International Conference on Information Processing in Medical Imaging, Springer. pp. 675-686.

Girard, G., Whittingstall, K., Deriche, R., Descoteaux, M., 2014. Towards quantitative connectivity analysis: reducing tractography biases. Neuroimage 98, 266-278.

Guevara, P., Poupon, C., Rivière, D., Cointepas, Y., Descoteaux, M., Thirion, B., Mangin, J.F., 2011. Robust clustering of massive tractography datasets. NeuroImage 54, 1975-1993.

Hau, J., Sarubbo, S., Houde, J.C., Corsini, F., Girard, G., Deledalle, C., Crivello, F., Zago, L., Mellet, E., Jobard, G., et al., 2017. Revisiting the human uncinate fasciculus, its subcomponents and asymmetries with stem-based tractography and microdissection validation. Brain Structure and Function 222, 1645-1662.

Hau, J., Sarubbo, S., Perchey, G., Crivello, F., Zago, L., Mellet, E., Jobard, G., Joliot, M., Mazoyer, B.M., Tzourio-Mazoyer, N., et al., 2016. Cortical terminations of the inferior fronto-occipital and uncinate fasciculi: anatomical stem-based virtual dissection. Frontiers in neuroanatomy $10,58$.

Jbabdi, S., Johansen-Berg, H., 2011. Tractography: where do we go from here? Brain connectivity 1, 169-183.

Jeurissen, B., Descoteaux, M., Mori, S., Leemans, A., 2017. Diffusion mri fiber tractography of the brain. NMR in Biomedicine.

Jeurissen, B., Leemans, A., Tournier, J.D., Jones, D.K., Sijbers, J., 2013. Investigating the prevalence of complex fiber configurations in white matter tissue with diffusion magnetic resonance imaging. Human brain mapping 34, 2747-2766.

Jones, D.K., 2010. Challenges and limitations of quantifying brain connectivity in vivo with diffusion mri. Imaging in Medicine 2, 341.

Kreher, B., Mader, I., Kiselev, V., 2008. Gibbs tracking: a novel approach for the reconstruction of neuronal pathways. Magnetic Resonance in Medicine 60, 953-963.

Kurtzer, G.M., Sochat, V., Bauer, M.W., 2017. Singularity: Scientific containers for mobility of compute. PloS one 12, e0177459.

Maier-Hein, K.H., Neher, P.F., Houde, J.C., Côté, M.A., Garyfallidis, E., Zhong, J., Chamberland, M., Yeh, F.C., Lin, Y.C., Ji, Q., et al., 2017. The challenge of mapping the human connectome based on diffusion tractography. Nature communications 8, 1349

Mangin, J.F., Fillard, P., Cointepas, Y., Le Bihan, D., Frouin, V., Poupon, C., 2013. Toward global tractography. Neuroimage 80, 290-296.

Mazoyer, B., Mellet, E., Perchey, G., Zago, L., Crivello, F., Jobard, G., Delcroix, N., Vigneau, M., Leroux, G., Petit, L., et al., 2016. Bil\&gin: a neuroimaging, cognitive, behavioral, and genetic database for the study of human brain lateralization. Neuroimage 124, 1225-1231.

Meola, A., Comert, A., Yeh, F.C., Stefaneanu, L., Fernandez-Miranda, J.C., 2015. The controversial 
existence of the human superior fronto-occipital fasciculus: Connectome-based tractographic study with microdissection validation. Human brain mapping 36, 4964-4971.

Mori, S., Crain, B.J., Chacko, V.P., Van Zijl, P.C., 1999. Three-dimensional tracking of axonal projections in the brain by magnetic resonance imaging. Annals of Neurology: Official Journal of the American Neurological Association and the Child Neurology Society 45, 265-269.

Mori, S., Wakana, S., Van Zijl, P.C., Nagae-Poetscher, L., 2005. MRI atlas of human white matter. Elsevier.

Mori, S., van Zijl, P., 2002. Fiber tracking: principles and strategies-a technical review. NMR in Biomedicine 15, 468-480.

Moyer, D., Thompson, P.M., Steeg, G.V., 2018. Measures of tractography convergence. arXiv preprint arXiv:1806.04634

Neher, P.F., Stieltjes, B., Reisert, M., Reicht, I., Meinzer, H.P., Fritzsche, K.H., 2012. Mitk global tractography, in: Medical Imaging 2012: Image Processing, International Society for Optics and Photonics. p. 83144D.

O'donnell, L.J., Golby, A.J., Westin, C.F., 2013. Fiber clustering versus the parcellation-based connectome. NeuroImage 80, 283-289.

Pestilli, F., Yeatman, J.D., Rokem, A., Kay, K.N., Wandell, B.A., 2014. Evaluation and statistical inference for human connectomes. Nature methods 11, 1058.

Portegies, J.M., Fick, R.H.J., Sanguinetti, G.R., Meesters, S.P., Girard, G., Duits, R., 2015. Improving fiber alignment in hardi by combining contextual pde flow with constrained spherical deconvolution. PloS one 10, e0138122.

Raffelt, D.A., Tournier, J.D., Smith, R.E., Vaughan, D.N., Jackson, G., Ridgway, G.R., Connelly, A., 2017. Investigating white matter fibre density and morphology using fixel-based analysis. Neuroimage $144,58-73$.

Reisert, M., Kiselev, V.G., 2011. Fiber continuity: an anisotropic prior for odf estimation. IEEE transactions on medical imaging 30, 1274-12 83.

Reisert, M., Mader, I., Anastasopoulos, C., Weigel, M., Schnell, S., Kiselev, V., 2011. Global fiber reconstruction becomes practical. Neuroimage 54, 955-962.

Renauld, E., Descoteaux, M., Bernier, M., Garyfallidis, E., Whittingstall, K., 2016. Semi-automatic segmentation of optic radiations and lgn, and their relationship to eeg alpha waves. PloS one 11 , $\mathrm{e} 0156436$

Rheault, F., Houde, J.C., Descoteaux, M., 2017. Visualization, interaction and tractometry: Dealing with millions of streamlines from diffusion mri tractography. Frontiers in neuroinformatics 11, 42.

Rheault, F., Roy, M., Cunnane, S., Descoteaux, M., 2018. Bundle-specific fornix reconstruction for dual-tracer pet-tractometry. bioRxiv , 423459.

Rozanski, V.E., da Silva, N.M., Ahmadi, S.A., Mehrkens, J., da Silva Cunha, J., Houde, J.C., Vollmar, C., Bötzel, K., Descoteaux, M., 2017. The role of the pallidothalamic fibre tracts in deep brain stimulation for dystonia: a diffusion mri tractography study. Human brain mapping 38, 1224-1232.

Smith, R.E., Tournier, J.D., Calamante, F., Connelly, A., 2012. Anatomically-constrained tractography: improved diffusion mri streamlines tractography through effective use of anatomical information. Neuroimage 62, 1924-1938.

Smith, R.E., Tournier, J.D., Calamante, F., Connelly, A., 2015a. The effects of sift on the reproducibility and biological accuracy of the structural connectome. Neuroimage 104, 253-265.

Smith, R.E., Tournier, J.D., Calamante, F., Connelly, A., 2015b. Sift2: Enabling dense quantitative assessment of brain white matter connectivity using streamlines tractography. Neuroimage 119, 338351.

Song, J.W., Mitchell, P.D., Kolasinski, J., Ellen Grant, P., Galaburda, A.M., Takahashi, E., 2014. Asymmetry of white matter pathways in developing human brains. Cerebral cortex 25, 2883-2893.

Sotiropoulos, S.N., Zalesky, A., 2017. Building connectomes using diffusion mri: Why, how and but. NMR in Biomedicine .

St-Onge, E., Daducci, A., Girard, G., Descoteaux, M., 2017. Surface-enhanced tractography (set). NeuroImage.

Tournier, J., Calamante, F., Connelly, A., et al., 2012. Mrtrix: diffusion tractography in crossing fiber regions. International Journal of Imaging Systems and Technology 22, 53-66.

Tournier, J.D., Yeh, C.H., Calamante, F., Cho, K.H., Connelly, A., Lin, C.P., 2008. Resolving crossing fibres using constrained spherical deconvolution: validation using diffusion-weighted imaging phantom data. Neuroimage 42, 617-625.

Voineskos, A.N., O'donnell, L.J., Lobaugh, N.J., Markant, D., Ameis, S.H., Niethammer, M., Mulsant, B.H., Pollock, B.G., Kennedy, J.L., Westin, C.F., et al., 2009. Quantitative examination of a novel 
clustering method using magnetic resonance diffusion tensor tractography. Neuroimage 45, 370-376.

Wang, R., Benner, T., Sorensen, A.G., Wedeen, V.J., 2007. Diffusion toolkit: a software package for diffusion imaging data processing and tractography, in: Proc Intl Soc Mag Reson Med, Berlin.

Wassermann, D., Makris, N., Rathi, Y., Shenton, M., Kikinis, R., Kubicki, M., Westin, C.F., 2016. The white matter query language: a novel approach for describing human white matter anatomy. Brain Structure and Function 221, 4705-4721.

Wasserthal, J., Neher, P., Maier-Hein, K.H., 2018a. Tractseg-fast and accurate white matter tract segmentation. arXiv preprint arXiv:1805.07103.

Wasserthal, J., Neher, P.F., Maier-Hein, K.H., 2018b. Tract orientation mapping for bundle-specific tractography. arXiv preprint arXiv:1806.05580.

Woolrich, M.W., Jbabdi, S., Patenaude, B., Chappell, M., Makni, S., Behrens, T., Beckmann, C., Jenkinson, M., Smith, S.M., 2009. Bayesian analysis of neuroimaging data in fsl. Neuroimage 45, S173-S186.

Yeatman, J.D., Dougherty, R.F., Myall, N.J., Wandell, B.A., Feldman, H.M., 2012. Tract profiles of white matter properties: automating fiber-tract quantification. PloS one 7, e49790.

Yeatman, J.D., Richie-Halford, A., Smith, J.K., Keshavan, A., Rokem, A., 2018. A browser-based tool for visualization and analysis of diffusion mri data. Nature communications 9, 940.

Yeh, F.C., Tseng, W.Y.I., 2011. Ntu-90: a high angular resolution brain atlas constructed by q-space diffeomorphic reconstruction. Neuroimage 58, 91-99.

Yendiki, A., Panneck, P., Srinivasan, P., Stevens, A., Zöllei, L., Augustinack, J., Wang, R., Salat, D., Ehrlich, S., Behrens, T., et al., 2011. Automated probabilistic reconstruction of white-matter pathways in health and disease using an atlas of the underlying anatomy. Frontiers in neuroinformatics 5, 23.

Zhang, F., Wu, W., Ning, L., McAnulty, G., Waber, D., Gagoski, B., Sarill, K., Hamoda, H.M., Song, Y., Cai, W., et al., 2018. Suprathreshold fiber cluster statistics: Leveraging white matter geometry to enhance tractography statistical analysis. NeuroImage .

Zhang, Y., Zhang, J., Oishi, K., Faria, A.V., Jiang, H., Li, X., Akhter, K., Rosa-Neto, P., Pike, G.B. Evans, A., et al., 2010. Atlas-guided tract reconstruction for automated and comprehensive examination of the white matter anatomy. Neuroimage 52, 1289-1301. 


\section{Appendix A. Anatomical Definition}

The PT was extracted following the anatomical definition proposed by Chenot et al. [2018] based on a precise manual positioning of individual subcortical regions of interest (ROI) along the descending pathway of the PT. Three ROIs were therefore drawn in each hemisphere within the internal capsule, the midbrain and the medulla oblongata using both b0 and color FA maps. This was performed with TrackVis [Wang et al., 2007]. Additional anatomical ROIs were used from the JHU template [Zhang et al., 2010] once warped to the native diffusion space of each subject using ANTS non-linear registration. Streamlines passing through the 3 ROIs, terminating either in the frontal lobe or the parietal lobe, and not terminating in the cerebellum were considered to compose the PT.

The AF was extracted in each hemisphere from a first set of streamlines with one termination in the frontal lobe and the other either in the temporal lobe or in the occipital lobe. Then, streamlines that do not pass through the ventral part of the external capsule (vEC) were considered as composing the AF. Such streamlines are anatomically constraint to leave the frontal lobe from the superior part of the external capsule and then present an arcuate shape defining the AF. The vEC ROI was composed of the region of the JHU template located in the ventral part of the external capsule, namely the inferior fronto-occipital and uncinate ROIs. Note that frontal, temporal and occipital lobes were composed of the frontal, temporal and occipital cortical gray matter and superficial white matter regions of the JHU template, respectively.

The CC was extracted from a first set of commissural streamlines with one termination in each hemisphere. A precentral-postcentral ROI, composed of the gray matter and superficial white matter part of the precentral and postcentral gyri of the JHU template was created for each hemisphere. Then, the present CC bundle was composed of callosal streamlines with one termination in each of the precentral-postcentral ROI and passing through the corpus callosum.

It is important to mention that streamlines considered for segmentation come from a tractogram generated with certain constraints and conditions. All streamlines are within the subject white matter, respect the length threshold $(20 \mathrm{~mm}-200 \mathrm{~mm})$, the angular threshold (45 degrees cone aperture for probabilistic and 20 degrees cone aperture for deterministic) and the minimum relative spherical function threshold of 0.1 ) 


\section{Appendix B. Annexes}

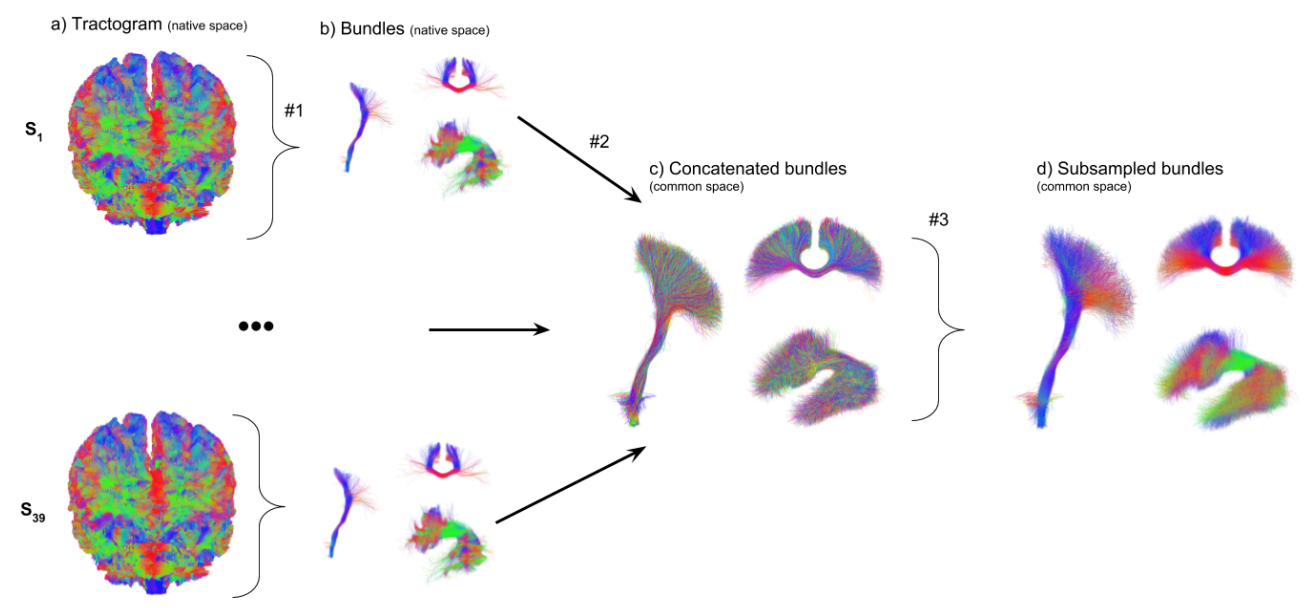

Figure B.9: Overview of the template creation pipeline. From whole brain tractogram (a) bundles are extracted (b) and then warped and concatenated in a common space (c), finally the density bias is reduced by a spatial subsampling (d)

\section{Appendix B.2. Results from all tractography algorithms}

In this section results from all tractography type are shown, comparaison to global tractography from the Medical Imaging Toolkit (MITK) [Neher et al., 2012; Kreher et al., 2008] was added. The tracking was performed with the original FOD and WM mask, default parameters and $1 \times 10^{8}$ iterations. For a fair comparaison, each subject whole brain tractogram was filtered to be similar to the classic tractography. Tractogram was forced to end in the BOI adapted interface, subsampled to the same number of streamlines and then segmented using the same automatic method. 

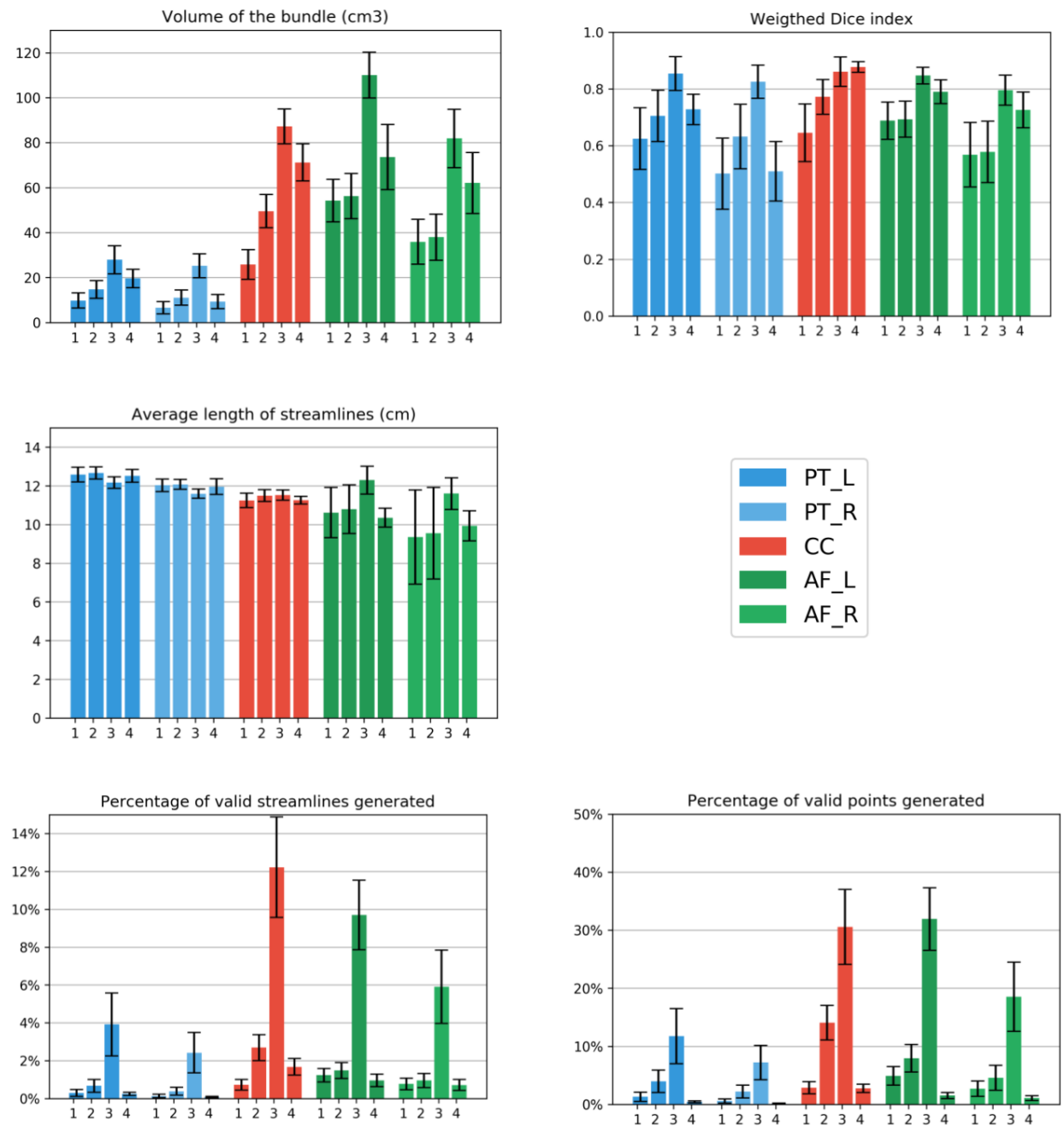

Figure B.10: Results for each BOI across the 4 tracking variations with probabilistic particle filtering tractography, where the fourth method is the global tracking. 

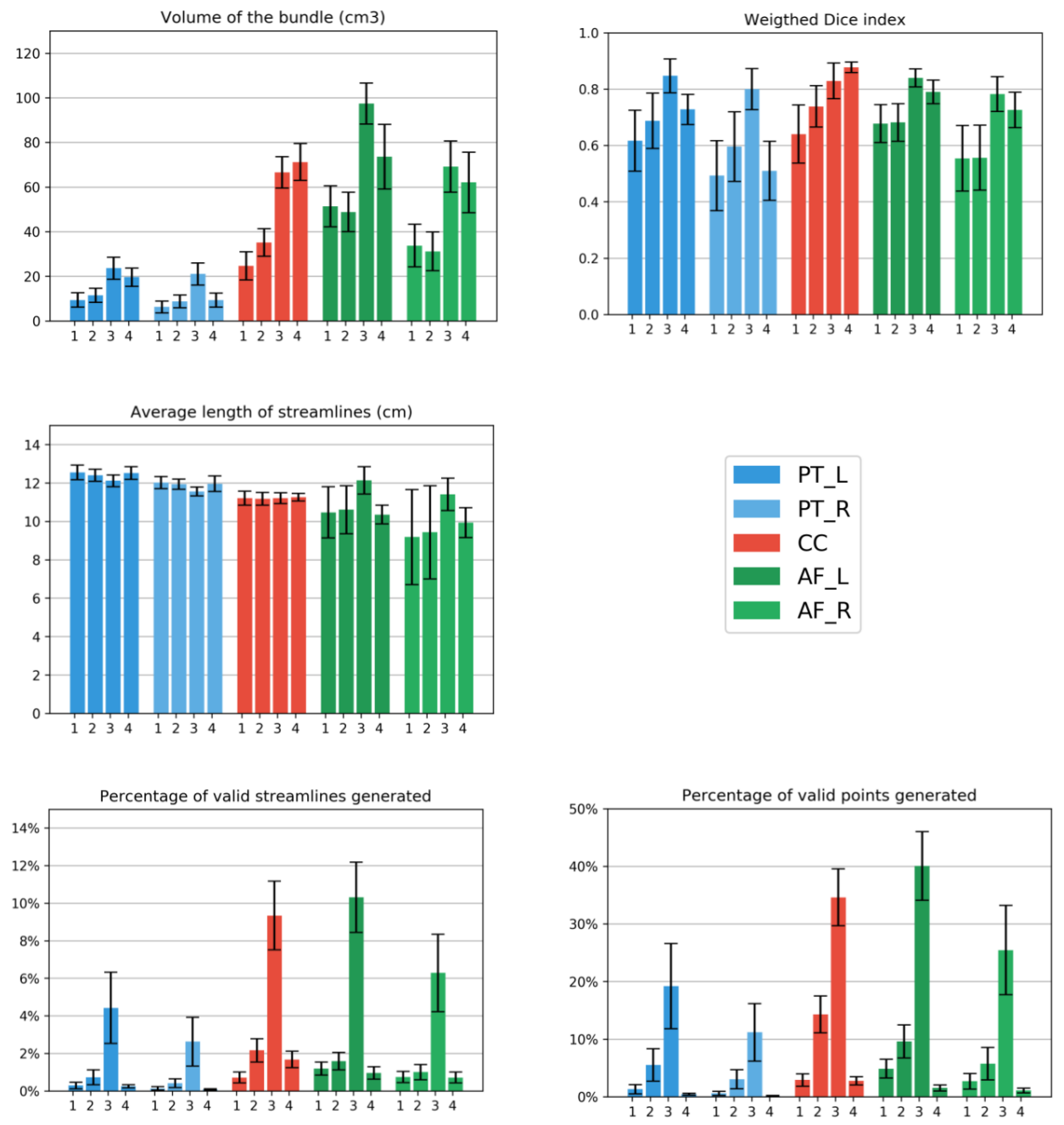

Figure B.11: Results for each BOI across the 4 tracking variations with probabilistic tractography, where the fourth method is the global tracking. 

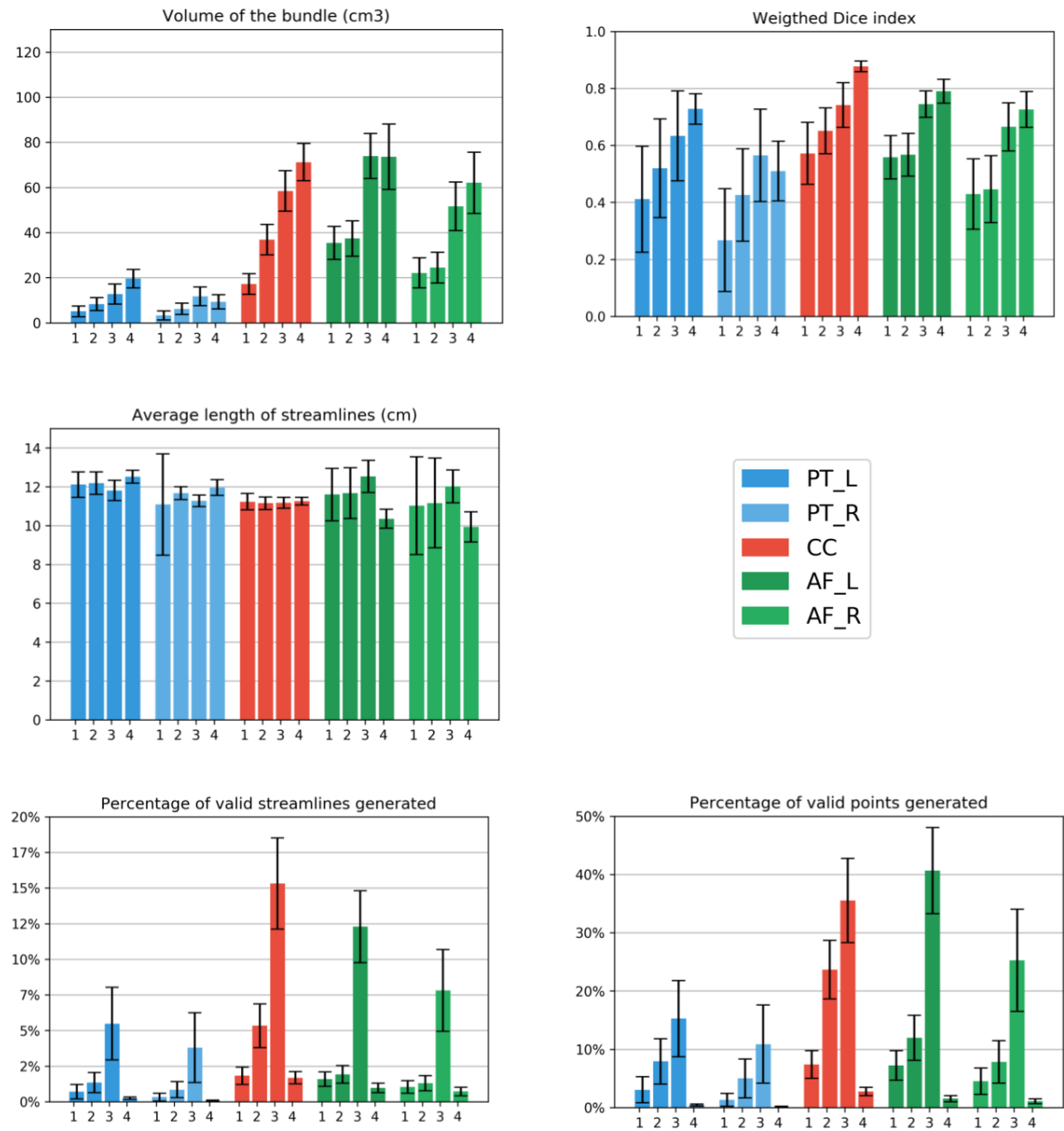

Figure B.12: Results for each BOI across the 4 tracking variations with deterministic particle filtering tractography, where the fourth method is the global tracking. 

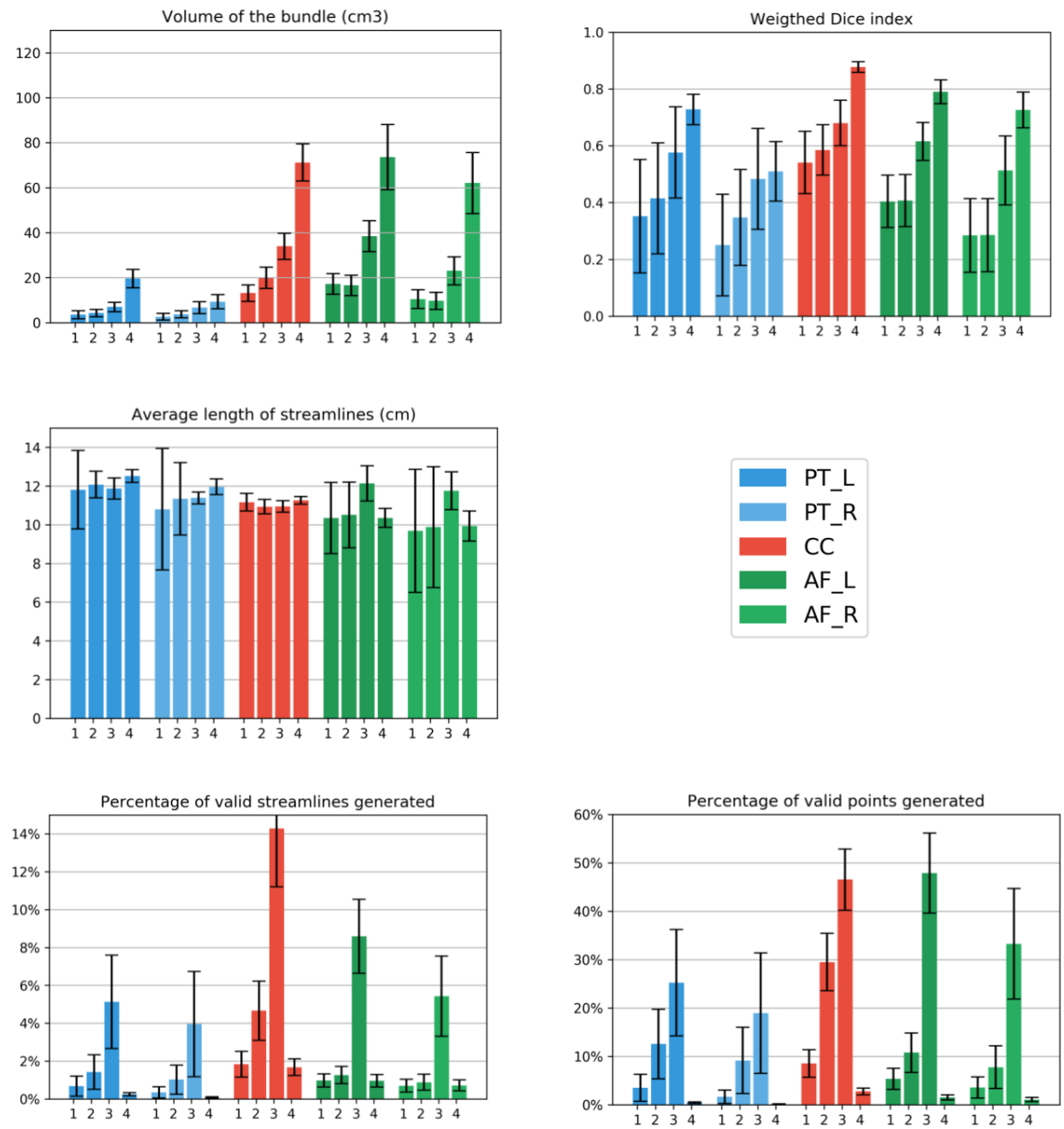

Figure B.13: Results for each BOI across the 3 tracking variations with deterministic tractography, where the fourth method is the global tracking. 